\title{
A method for determining the average height of a large topographic ice sheet from observations of the echo received by a satellite altimeter
}

\author{
D. J. WINGHAM \\ Mullard Space Science Laboratory, University College London, Holmbury St. Mary, Dorking, Surrey RH5 6NT, England
}

\begin{abstract}
Ice-sheet surfaces have scales of fluctuation that are similar to the diameter of the area illuminated by a satellite radar altimeter. The present theory of altimetry, developed to describe scattering from the ocean surface, does not deal properly with the geometry of ice-sheet surfaces. In this paper, the theory of altimetry is extended to cover this geometry. A general relation for the altimeter echo from a surface of unknown geometry is developed, including the effects of the penetration of the surface by the radar waves. This expression is linearized, using the characteristic operating geometry of satellite altimeters and the gentle nature of ice-sheet gradients. From this expression, an integral equation is derived, from whose solution the spatial average of the height of the surface relative to a spherical datum can be determined. The integral equation is of a Volterra type, which permits the uniqueness of the solution for the average height to be investigated simply. The method is extended to provide a solution for the spatial average of the height of a local region of the ice sheet, provided the region remains large in comparison with the area illuminated by the altimeter, and to deal with variations in the antenna boresight alignment. The results have a number of implications for the collection and reduction of echoes in an experiment to determine the average height of an ice sheet. The unique determination of the average height requires the echo to be known over a time interval that depends on the extrema of the surface, which therefore must be known a priori. The average height itself can be determined by the operation on the echo of a linear operator whose kernel is derived from the solution of the Volterra-type equation. This marks a change from the procedures currently used in practice to reduce echoes from ice sheets.
\end{abstract}

\section{LIST OF SYMBOLS}

$A$

$c$

$c_{\mathrm{i}}$

C

$f$

$f_{0}$

$f_{1}$

$f_{\mathrm{s}}$

$\bar{f}$

$\bar{f}_{\mathrm{W}}$

$g$

$h$

$h_{0}$

$H$

$I$ sphere
Area of the projection of the ice surface on to the reference sphere

Velocity of propagation in air

Velocity of propagation in ice

See Equation (58)

Height of the surface above the reference

Maximum height of the surface above the reference sphere

Minimum height of the surface above the reference sphere

See Equation (95)

Average height of the surface above the reference sphere

Weighted average height of the surface above the reference sphere

Altimeter power gain

Satellite altitude above the reference sphere

A constant satellite altitude above the reference sphere

Location of the satellite

See Equation (44)

\begin{tabular}{|c|c|}
\hline$I_{\mathrm{W}}$ & See Equation (63) \\
\hline$I_{\mathrm{WL}}$ & See Equation (73) \\
\hline$I_{0}$ & $\begin{array}{l}\text { Bessel function of imaginary argument } \\
\text { (Abramowitz and Stegun, 1965, section 9.6) }\end{array}$ \\
\hline$I_{1}$ & See Equation (86) \\
\hline$j$ & See Equation (54) \\
\hline$j_{\mathrm{WL}}$ & See Equation (87) \\
\hline$J$ & See Equation (88) \\
\hline$J_{\mathrm{WL}}$ & See paragraph following Equation $(90)$ \\
\hline$k$ & See Equation (49) \\
\hline$k_{1}$ & Extinction coefficient of ice \\
\hline$k_{\mathrm{t}}$ & Power-transmission loss at interface \\
\hline$L(\ell)$ & Power-transmission loss along a path length $\ell$ \\
\hline$p$ & Echo power \\
\hline$p_{\mathrm{a}}$ & Time-advanced echo power \\
\hline$p_{\mathrm{i}}$ & Incident-power density \\
\hline$\hat{p}$ & See Equation (48) \\
\hline$\hat{p}_{\mathrm{W}}$ & See Equation (61) \\
\hline$p_{\delta \mathrm{A}}$ & $\begin{array}{l}\text { Echo-power density at the altimeter antenna } \\
\text { from a surface element }\end{array}$ \\
\hline$p_{\delta \mathrm{V}}$ & $\begin{array}{l}\text { Echo-power density at the altimeter antenna } \\
\text { from a volume element }\end{array}$ \\
\hline 1 & Transmitted power envelope \\
\hline & See Equation (37) \\
\hline
\end{tabular}


$\bar{q}_{\mathrm{W}} \quad$ See Equation $(60)$

$r(\mathrm{~A}, \mathrm{~B}) \quad$ Distance between two points $\mathrm{A}$ and $\mathrm{B}$

$R \quad$ Radius of the reference sphere

$\hat{s} \quad$ See Equation (55)

$t \quad$ Time

$t_{0} \quad$ See paragraph succeeding Equation (50)

$t_{1} \quad$ See paragraph succeeding Equation (50)

$T_{\mathrm{p}} \quad$ Duration of transmitted power envelope

$T_{0} \quad$ Minimum time for which the advanced echo is known

$T_{1} \quad$ Maximum time for which the advanced echo

is known

$\hat{u} \quad$ See Equation (89)

$\bar{x}, \bar{x}_{1}, \bar{x}_{2}, \bar{x}_{3}$ Solutions of Equation (51)

$\bar{y} \quad$ Solution of Equation (53)

$z \quad$ Elevation measured normally to the reference sphere

$\delta A \quad$ Area of a surface element

Sh The departure of the satellite altitude from the constant altitude $h_{0}$

$\delta S, \delta S^{\prime}, \delta S^{\prime \prime}$ See paragraph preceding Equation (23)

$\delta V \quad$ Volume of an element of ice

$\Delta \quad$ Angle between tangent planes of the local and geocentric spheres

$\phi(\mathrm{A}, \mathrm{B}) \quad$ The angle subtended by points $\mathrm{A}$ and $\mathrm{B}$ at the centre of the reference sphere

$\phi_{\mathrm{W}} \quad$ Angular width of the weighting function

$\theta(\mathrm{H}, \mathrm{A}) \quad$ The angle subtended at the altimeter by the point $\mathrm{A}$ and the antenna boresight

$\Theta \quad$ See Equation (77)

$\lambda \quad$ Transmitted carrier wavelength

$\sigma^{0} \quad$ Surface back-scattering coefficient

$\sigma_{\nu} \quad$ Volume back-scattering coefficient

$\nabla \quad$ Angle between the ice surface and the tangent plane to the reference sphere

$\eta \quad$ See Equation (20)

$\kappa \quad$ See Equation (76)

\section{INTRODUCTION}

The mapping of the ice sheets and ice shelves of Antarctica and Greenland by satellite altimetry has, for the past 15 years, exploited measurements from an experimental design developed in the $1970 \mathrm{~s}$ to determine the shape of the marine geoid (McGoogan, 1975). This design arose from the theoretical understanding, developed at the time and brought to a closed form by Brown (1977), of the shape of the altimeter echo from the ocean surface. This understanding allowed the mean height of the ocean surface to be determined uniquely from a truncated measurement of the echo. It has long been recognized (see e.g. Robin and others, 1983) that the geometric differences between the shape of the geoid and the shape of an ice sheet introduce a uniqueness problem in extending the experimental method to ice sheets. However, no extension to the theory of altimetry has been given that would permit the problem to be closed in theory, nor has any usefully general description been given of what assumptions are needed to close the problem in practice.

The theory of Brown (1977) requires the surface to have two widely separated scales of surface undulation. The diameter of the area illuminated by the altimeter is assumed much smaller than the larger scale and much larger than the smaller scale. The smaller-scale undulations, termed roughness, are assumed to have a known probability density function. The larger-scale undulation can then be regarded as the ensemble mean of the roughness, which is small enough in scale that its effect on the altimeter echo can be taken to equal the ensemble mean echo. Ice sheets, however, have topography over a wide range of spatial scales which includes the diameter of the area illuminated by the altimeter. In this circumstance, statistical arguments are of limited use, because the interchange of a spatial average and an ensemble average is no longer a simple matter.

The purpose of this paper is to extend the theory of altimetry to deal with topographic surfaces, without depending on statistical arguments or constraints on the scale of undulation. In particular, we seek to answer the following four questions: What is the relation between the altimeter echo and the height of a topographic surface? Can a method be found for the inversion of this relationship for the average height? In what circumstances is the result of this inversion unique? What do the answers to these questions imply for the design of an experiment to determine the average height of ice sheets?

In section 3, the first of these questions is dealt with. A general expression for the echo from a topographic surface is given, including the effects of the radar penetration of the surface discovered by Ridley and Partington (1988). The surface height, relative to a spherical datum, enters this expression in an extremely complicated way and the remainder of the section is devoted to using the special character of satellitealtimeter geometry to simplify the height-dependence of the echo. In section 4, the spatial average of the height is introduced. The task of determining it from the echo is reduced in section 4.1 to solving an integral equation with a Volterra kernel. The form of the kernel allows us to deal simply in section 4.2 with the issue of uniqueness, which turns out to depend on the echo gating. In section 4.3 we show how simple modifications allow the area over which the average is formed to be varied. These results are restricted to the case where the antenna boresight is maintained normal to the datum sphere, so in section 4.4 we lift this restriction. In section 5 , the implications for the collection and reduction of altimeter echoes are discussed, we comment on the methods of reduction employed presently in practical reconstruction schemes and we summarize the limitations of the method. Finally, in section 6 we present some conclusions.

The results of this work should find their most important practical application in helping to constrain the mass balances of the large ice sheets of Antarctica and Greenland, where it is the variation with time in the height averaged over large length scales that is principally of interest. We do, in passing, consider the determination of the height itself. However, one of the results of this paper is that the determination of the height is a more complicated problem which deserves a separate treatment in its own right. 


\section{THE FORWARD PROBLEM}

The problem we consider in this paper is to determine the average height of an ice sheet from observations of the radar echo scattered from its surface. This problem is in two parts. The first part, the forward problem, is to form an integral expression for the echo. The second part, the inverse problem, is to solve the integral expression for the average height. The forward problem is the task of this section; the inverse problem is dealt with in section 4 .

Inverse problems are generally very much simpler if they can be linearized. The main task of this section is to give approximate forms for the echo that allow us to approach the inverse problem within a linear framework. We start by giving a general expression for the echo from an ice sheet that contributes surface- and volume-scatter to the echo. We then make a number of approximations to arrive at a form suitable for linear inversion.

\subsection{The altimeter echo; the basis for its approximation}

The general arrangement is shown in Figure 1. An altimeter at the point $\mathrm{H}$ transmits a pulse $q(t)$ of duration $T_{\mathrm{p}}$. (A list of symbols is given in section 1.) The pulse travels out towards the surface at velocity $c$, occupying at any time a spherical shell of radius $c t$ and thickness $c T_{\mathrm{p}}$. The pulse amplitude is modulated by the antenna-gain pattern $g(\sin (\theta))$, where $\theta$ is the angle subtended by the antenna boresight and a line joining the point $\mathrm{H}$ to a point on the shell. The power per unit area $p_{\mathrm{i}}(t)$ incident at a point $\mathrm{P}$ on the air-ice interface a path length $r(\mathrm{H}, \mathrm{P})$ from $\mathrm{H}$ is

$$
p_{\mathrm{i}}(\mathrm{H}, \mathrm{P}, t)=\frac{g(\sin (\theta(\mathrm{H}, \mathrm{P}))) q(t-r(\mathrm{H}, \mathrm{P}) / c)}{4 \pi r^{2}(\mathrm{H}, \mathrm{P})} .
$$

There are two contributions to the echo scattered back to the altimeter. One is a contribution from the air-ice interface. This is modelled with a surface back-scattering coefficient $\sigma^{0}$, such that the power per unit area incident at $\mathrm{H}$, scattered from an area element $\delta A$ of the surface, is

$$
\begin{aligned}
p_{\delta \mathrm{A}}(\mathrm{H}, \mathrm{P}, t) & =\frac{\sigma^{0} p_{\mathrm{i}}(\mathrm{H}, \mathrm{P}, t-r(\mathrm{H}, \mathrm{P}) / c) \delta A}{4 \pi r^{2}(\mathrm{H}, \mathrm{P})} \\
& =\frac{\sigma^{0} g(\sin (\theta(\mathrm{H}, \mathrm{P}))) q(t-2 r(\mathrm{H}, \mathrm{P}) / c) \delta A}{(4 \pi)^{2} r^{4}(\mathrm{H}, \mathrm{P})}
\end{aligned}
$$

where the second line uses Equation (1).

The second contribution to the echo comes from energy that penetrates the interface and is scattered from a volume element around a point $\mathrm{Q}$ within the volume of the ice. Before reaching the point $Q$, the incident energy is first reduced by a factor $k_{\mathrm{t}}$ in passing the interface at $\mathrm{P}^{\prime}$. Since, later, we shall assume all angles of incidence are small, we shall neglect refraction at the interface and, in this case, $\mathrm{P}^{\prime}$ is the point of intersection of the line $\mathrm{HQ}$ and the surface. Within the volume of the ice, the pulse travels at a velocity $c_{\mathrm{i}}$. Travelling within the ice, the pulse is further reduced by losses $L$ in traversing the path length $r\left(\mathrm{P}^{\prime}, \mathrm{Q}\right)$. To model the loss experienced along a path length $\ell$, we follow Ridley and Partington (1988), and use an exponential decay law

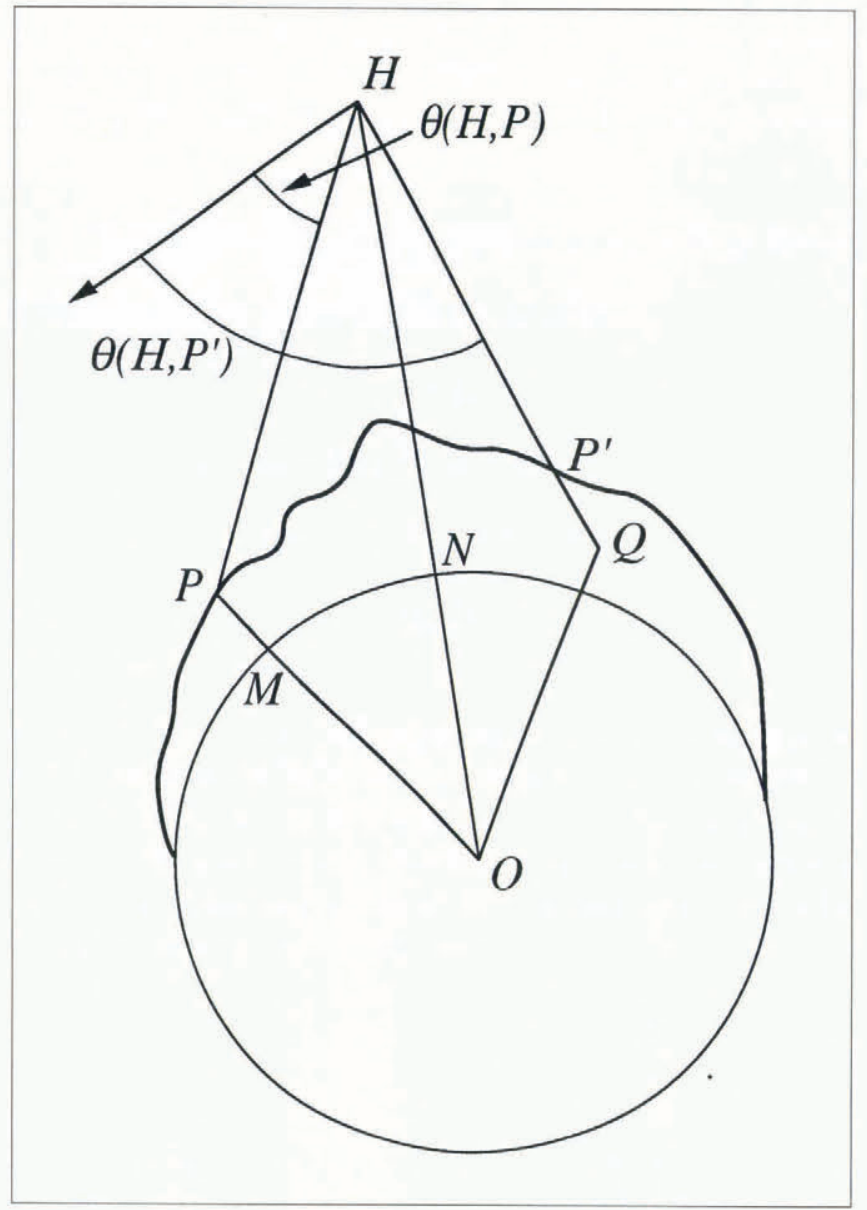

Fig. 1. The geometry of a radar-altimeter measurement of an ice sheet. Power transmitted from an altimeter at the point $\mathrm{H}$ receives power scattered from a point $\mathrm{P}$ on the surface of the ice sheet. Power reaching $\mathrm{P}$ is reduced by the inverse square of the path length $r(\mathrm{H}, \mathrm{P})$ and power scattered back to the altimeter suffers a further reduction by the same factor. Power entering the ice is scattered from a point $\mathrm{Q}$ within the body of the ice. In reaching $\mathrm{Q}$ from the point $\mathrm{P}^{\prime}$ and returning to the point $\mathrm{P}^{\prime}$, the power suffers an additional attenuation that is a function of the path length $r\left(\mathrm{P}^{\prime}, \mathrm{Q}\right)$. The power received by the altimeter is modulated by the antenna gain of the altimeter, which is a function of the angle $\theta$ subtended by the direction of the antenna boresight and the line joining $\mathrm{H}$ to the scattering point. The points $\mathrm{O}, \mathrm{N}$ and $\mathrm{M}$ are described in the text. Note that the geometry is shown grossly distorted for clarity.

$$
L(\ell)=\exp \left(-k_{1} \ell\right)
$$

where $k_{1}$ is termed the extinction coefficient. Assuming that the interaction at the surface is weak enough that forward scatter from the surface can be ignored, the power per unit area incident at the point $\mathrm{Q}$ is

$$
\begin{aligned}
p_{\mathrm{i}}(\mathrm{H}, \mathrm{Q}, t)=( & g\left(\sin \left(\theta\left(\mathrm{H}, \mathrm{P}^{\prime}\right)\right)\right) k_{\mathrm{t}} \exp \left[-k_{1} r\left(\mathrm{P}^{\prime}, \mathrm{Q}\right)\right] \\
& \left.\cdot q\left(t-r\left(\mathrm{H}, \mathrm{P}^{\prime}\right) / c-r\left(\mathrm{P}^{\prime}, \mathrm{Q}\right) / c_{\mathrm{i}}\right)\right) \\
& /\left(4 \pi r^{2}(\mathrm{H}, \mathrm{Q})\right) .
\end{aligned}
$$

The scattering from the element around the point $\mathrm{Q}$ is modelled with a volume back-scattering coefficient $\sigma_{\nu}$. 
Energy scattered from $\mathrm{Q}$ and received at the altimeter returns along the path $\mathrm{HP}^{\prime} \mathrm{Q}$. The power per unit area incident at $\mathrm{H}$, scattered from a volume element $\delta V$ at $\mathrm{Q}$, is

$$
\begin{aligned}
p_{\delta \mathrm{V}}(\mathrm{H}, \mathrm{Q}, t)= & \left(\sigma_{\nu} g\left(\sin \left(\theta\left(\mathrm{H}, \mathrm{P}^{\prime}\right)\right)\right) k_{\mathrm{t}} \exp \left[-k_{1} r\left(\mathrm{P}^{\prime}, \mathrm{Q}\right)\right]\right. \\
& \left.\cdot p_{\mathrm{i}}\left(\mathrm{H}, \mathrm{Q}, t-r\left(\mathrm{H}, \mathrm{P}^{\prime}\right) / c-r\left(\mathrm{P}^{\prime}, \mathrm{Q}\right) / c_{\mathrm{i}}\right) \delta V\right) \\
& /\left(4 \pi r^{2}(\mathrm{H}, \mathrm{Q})\right) \\
= & \left(\sigma_{\nu} g\left(\sin \left(\theta\left(\mathrm{H}, \mathrm{P}^{\prime}\right)\right)\right) k_{\mathrm{t}}^{2} \exp \left[-2 k_{1} r\left(\mathrm{P}^{\prime}, \mathrm{Q}\right)\right]\right. \\
& \left.\cdot q\left(t-2\left(r\left(\mathrm{H}, \mathrm{P}^{\prime}\right) / c-r\left(\mathrm{P}^{\prime}, \mathrm{Q}\right) / c_{\mathrm{i}}\right)\right) \delta V\right) \\
& /\left((4 \pi)^{2} r^{4}(\mathrm{H}, \mathrm{Q})\right)
\end{aligned}
$$

where the second line uses Equation (4).

From antenna theory (Collin and Zucker, 1969), a power per unit area $p_{\mathrm{r}}$ incident on the antenna from a direction $\theta$ results in a received power $\lambda^{2} g(\sin \theta) p_{\mathrm{r}} /(4 \pi)$, where $\lambda$ is the transmitted carrier wavelength. The total received power $p$ is then the surface and volume contributions integrated respectively over the surface and volume of the ice:

$$
\begin{aligned}
p(\mathrm{H}, t)= & \frac{\lambda^{2}}{4 \pi}\left(\iint_{\text {Surface }} \mathrm{d} A g(\sin (\theta(\mathrm{H}, \mathrm{P}))) p_{\delta \mathrm{A}}(\mathrm{H}, \mathrm{P}, t)\right. \\
& \left.+\iiint_{\text {Volume }} \mathrm{d} V g\left(\sin \left(\theta\left(\mathrm{H}, \mathrm{P}^{\prime}\right)\right)\right) p_{\delta V}(\mathrm{H}, \mathrm{Q}, t)\right) \\
= & \frac{\lambda^{2}}{(4 \pi)^{3}}\left(\sigma^{0} \iint_{\text {Surface }} \mathrm{d} A\right. \\
& \cdot\left(g^{2}(\sin (\theta(\mathrm{H}, \mathrm{P}))) q(t-2 r(\mathrm{H}, \mathrm{P}) / c)\right) /\left(r^{4}(\mathrm{H}, \mathrm{P})\right) \\
& +\sigma_{\nu} k_{t}^{2} \iiint_{\text {Volume }} \mathrm{d} V\left(g^{2}\left(\sin \left(\theta\left(\mathrm{H}, \mathrm{P}^{\prime}\right)\right)\right)\right. \\
& \cdot \exp \left[-2 k_{1} r\left(\mathrm{P}^{\prime} \mathrm{Q}\right)\right] q\left(t-2\left(r\left(\mathrm{H}, \mathrm{P}^{\prime}\right) / c\right.\right. \\
& \left.\left.\left.-r\left(\mathrm{P}^{\prime}, \mathrm{Q}\right) / c_{\mathrm{i}}\right)\right)\right) \\
& \left./\left(r^{4}\left(\mathrm{H}^{2}, \mathrm{Q}\right)\right)\right) .
\end{aligned}
$$

We term $p$ the echo.

We will be concerned in section 4 to determine the average height of the point $\mathrm{P}$ above a sphere, from observations of the echo. For this purpose, we need to relate the quantities in Equation (6) to the sphere shown in Figure 1. Let the reference sphere have a radius $R$ with an origin at a point $\mathrm{O}$. We use a coordinate system such that any point $\mathrm{X}$ is then defined by the point $\mathrm{Y}$ lying at the intersection of the line OX and the sphere, and the height $z$ of the point $\mathrm{X}$, measured normal to the sphere.

Let $\mathrm{M}$ lie at the intersection of the line $\mathrm{OP}$ and the sphere. The surface is then defined by the function

$$
z=f(\mathrm{M}) \text {. }
$$

As it stands, $f(\mathrm{M})$, the height of the surface and the quantity of interest, enters Equation (6) in a very complicated fashion. To simplify its dependence in Equation (6), we shall make a number of geometric approximations, whose bases we now discuss.

Let $\mathrm{N}$ lie at the intersection of the line $\mathrm{OH}$ and the sphere as shown in Figure 1. Let $z=h(\mathrm{~N})$ describe the satellite altitude. For a radar-altimeter satellite, the altitude is typically $1000 \mathrm{~km}$. Ice-sheet topographies range, typically, over a few kilometres. We assume, therefore, that

$$
\frac{f}{h} \sim 10^{-3} .
$$

The antenna-gain pattern of satellite altimeters is chosen so that the power is appreciably non-zero only within a small range of angles subtended at $\mathrm{H}$, typically of order $1^{\circ}$. We will assume that the boresight lies close to the line $\mathrm{HO}$. In determining the power received at $\mathrm{H}$, one therefore need only consider points $\mathrm{P}$ for which the angle OHP is of the order of $1^{\circ}$. It will be convenient to express this in terms of the radian angle $\phi(N, M)$, subtended by the points $\mathrm{N}$ and $\mathrm{M}$ at $\mathrm{O}$. Specifically, we assume that

$$
\frac{R \sin \phi}{h} \sim 10^{-2}
$$

The radius $R$ of the reference sphere is not small. It is of the order of an Earth radius, about $10000 \mathrm{~km}$. We assume

$$
\frac{h}{R} \sim 10^{-1} .
$$

With Equation (9), this implies $\sin \phi \sim 10^{-3}$.

The radial component of the orbit of satellite altimeters does not vary greatly in comparison with the satellite altitude. In view of this, let $h(\mathrm{~N})$ be described by a perturbation $\delta h(\mathrm{~N})$ about a constant height $h_{0}$ above the sphere. We assume

$$
\frac{\delta h}{h_{0}} \sim 10^{-2}
$$

The magnitude of the extinction coefficient near the surface of ice sheets can vary considerably with carrier frequency and surface conditions but a typical path length will be of order $10 \mathrm{~m}$. We assume that

$$
h k_{1} \sim 10^{5}
$$

The surface gradients of ice sheets, relative to the tangent plane to the sphere, are small, rarely larger than a few degrees. Denoting this gradient by $\tan \nabla$, we assume that

$$
\tan \nabla \sim 10^{-1}
$$

The last characteristic scale that is important to us is 
the duration $T_{\mathrm{p}}$ of the pulse $q$. This is typically a few nanoseconds. With the observation that the wave speed is close to that of light in a vacuum, we assume

$$
\frac{h}{c T_{\mathrm{p}}} \sim 10^{6}
$$

In the theory we develop in this paper, we will ignore contributions to the integrands in Equation (6) that are $\mathrm{O}\left(10^{-2}\right)$ or smaller. In the next section, Equation (6) is approximated by truncating a series representation of the arguments of the functions forming the integrand. One needs, therefore, a method connecting the order of approximation of an argument to the order of approximation of a function. For this purpose, one may observe that, for some function $\nu(x)$, if one ignores a term $\delta x$ in its argument, one is ignoring a term $\nu^{\prime}(x) \delta x$ in the function to first order; to determine the order of the approximation to the integrand in Equation (6), then, one needs an approximation for the magnitudes of the functions $g^{\prime}$ and $q^{\prime}$. We shall take the view that, if a function $\nu(x)$ is appreciably non-zero over an extent $x_{\mathrm{e}}$, and, if it does not vary rapidly on scales of $x_{\mathrm{e}}$, then one may take $\nu(x) / x_{\mathrm{e}}$ as an approximation to the size of $\nu^{\prime}(x)$. This is a reasonable assumption for theoretical approximations to the functions that appear in Equation (6) in practical altimetry. Specifically, we assume for the function $g$ that

$$
\begin{aligned}
& g\left(\frac{R \sin \phi}{h}\left(1+\ldots \mathrm{O}\left(10^{-n}\right)\right)\right) \\
\sim & g\left(\frac{R \sin \phi}{h}(1+\ldots)\right)\left(1+\mathrm{O}\left(10^{-n}\right)\right)
\end{aligned}
$$

where we have used $(R / h) \sin \phi$ as the extent of $g$.

In the case of the pulse function $q$, one may use for its extent the duration $T_{\mathrm{p}}$. Our assumption is that

$$
\begin{aligned}
q(t- & \left.\left(\frac{2 h}{c}\right)\left(1+\ldots \mathrm{O}\left(10^{-n}\right)\right)\right) \\
& \sim q\left(t-\left(\frac{2 h}{c}\right)(1+\ldots)\right)\left(1+\mathrm{O}\left(\frac{h}{c T_{\mathrm{p}}} 10^{-n}\right)\right) \\
& =q\left(t-\left(\frac{2 h}{c}\right)(1+\ldots)\right)\left(1+\mathrm{O}\left(10^{-n+6}\right)\right)
\end{aligned}
$$

where the second line uses Equation (14).

Finally, for the exponential in Equation (6), we asume

$$
\begin{aligned}
\exp \left(-k_{1} h\right. & \left.\left(1+\ldots \mathrm{O}\left(10^{-n}\right)\right)\right) \\
& \sim \exp \left(-k_{1} h(1+\ldots)\right)\left(1+\mathrm{O}\left(k_{1} h 10^{-n}\right)\right) \\
& =\exp \left(-k_{1} h(1+\ldots)\right)\left(1+\mathrm{O}\left(10^{-n+5}\right)\right)
\end{aligned}
$$

where the second line uses Equation (12).

On comparing Equations (15), (16) and (17), one notices the fact that the integrand of Equation (6) is very much more sensitive to small changes to the ranges in the arguments of $q$ and the exponential than it is to small changes in the angle in the argument of $g$. This is characteristic of pulse-limited altimetry.

\subsection{The Fresnel and related approximations}

In this section, we simplify the way the height of the surface enters the integral in Equation (6).

Starting with the path length $r(\mathrm{H}, \mathrm{P})$, one has, on applying the cosine rule to the triangle OHP,

$$
\begin{aligned}
r(\mathrm{H}, \mathrm{P}) & =\left((R+h(\mathrm{~N}))^{2}+(R+f(\mathrm{M}))^{2}\right. \\
& -2(R+h(\mathrm{~N}))(R+f(\mathrm{M})) \cos (\phi(\mathrm{N}, \mathrm{M})))^{\frac{1}{2}}
\end{aligned}
$$

Expanding the square root in Equation (14), one has

$$
\begin{aligned}
r(\mathrm{H}, \mathrm{P})= & \\
& h_{0}\left(1+\frac{\delta h(\mathrm{~N})}{h_{0}}-\frac{f(\mathrm{M})}{h_{0}}+\frac{2 R^{2} \sin ^{2}(\phi(\mathrm{N}, \mathrm{M}) / 2)}{h_{0}^{2}}\right. \\
& \left.\cdot\left(1+\frac{h_{0}}{R}\right)+\mathrm{O}\left(10^{-8}\right)\right)
\end{aligned}
$$

The factor $1+h_{0} / R$ will appear repeatedly, and we introduce the notation

$$
\eta=1+h_{0} / R
$$

From Equation (10), it is apparent that $\eta$ is $\mathrm{O}(1)$.

For the terms in $r^{4}$ that occur in the denominator of Equation (6), one has, simply

$$
r^{4}=h_{0}^{4}\left(1+\mathrm{O}\left(10^{-2}\right)\right) .
$$

This, with Equation (19), constitutes the Fresnel approximation, widely used in the analysis of surface scattering (a good example is Berry (1973)), although not, to date, in altimetry theory.

We consider now the argument of the antenna-gain pattern $g$. This is a function only of $\sin \theta(\mathrm{H}, \mathrm{P})$. Later, we derive, correct to the lowest order, the approximation Equation (79) to $\sin \theta(\mathrm{H}, \mathrm{P})$ for a more general case which allows the antenna boresight to lie away from the normal to the sphere. In the case that the antenna boresight is normal to the sphere, this approximation simplifies to give

$$
\sin \theta(\mathrm{H}, \mathrm{P})=\frac{2 R}{h_{0}} \sin \left(\frac{\phi(\mathrm{N}, \mathrm{M})}{2}\right)\left(1+\mathrm{O}\left(10^{-4}\right)\right) .
$$

To deal with the area element $\delta A$ of the surface, let $\delta S$ be an element of area of the sphere containing the point M. Let $\delta S^{\prime}$ be the spherical projection of $\delta S$ on to a second sphere with origin $\mathrm{O}$ that passes through the point $\mathrm{P}$ on the surface. Let $\delta S^{\prime \prime}$ be the plane projection of $\delta S^{\prime}$ on to the tangent plane to the second sphere at $\mathrm{P} ; \tan \nabla$ is then the gradient of the surface with respect to this plane. Let $\delta A$ be the plane projection of $\delta S^{\prime \prime}$ on to the surface. Then, as the area element $\delta A$ tends to zero, 


$$
\begin{aligned}
\delta A \rightarrow & \sqrt{1+\tan ^{2} \nabla} \delta S^{\prime \prime} \rightarrow \sqrt{1+\tan ^{2} \nabla} \delta S^{\prime} \\
& =\frac{R^{2} \sqrt{1+\tan ^{2} \nabla} \delta S}{(R+f(\mathrm{M}))^{2}} \\
& =\delta S\left(1+\mathrm{O}\left(10^{-2}\right)\right) .
\end{aligned}
$$

The limits on the surface integral are now the spherical projection of the ice surface on to the sphere.

Turning now to the volume integral on the RHS of Equation (6), let $\mathrm{M}^{\prime \prime}$ lie at the intersection of the line $\mathrm{OQ}$ with the sphere, and let $\mathrm{M}^{\prime}$ lie at the intersection of the line $\mathrm{OP}^{\prime}$ with the sphere. This is shown in Figure 2. Let $z(\mathrm{Q})$ be the height of the point $\mathrm{Q}$ above $\mathrm{M}^{\prime \prime}$. Since $\mathrm{Q}$ lies within the volume, $z(\mathrm{Q}) \leq f\left(\mathrm{M}^{\prime \prime}\right)$. The volume integral is complicated by the fact that the location of the point $\mathrm{P}^{\prime}$ is an implicit function of the position of the point $\mathrm{Q}$ and the shape of the surface. To avoid this, we use below the gentle gradients of ice sheets to show that we can replace the point $\mathrm{P}^{\prime}$ with the point $\mathrm{P}^{\prime \prime}$, lying at the intersection of the line $\mathrm{OQ}$ and the surface. With this replacement, one then has

$$
\begin{aligned}
r\left(\mathrm{H}, \mathrm{P}^{\prime}\right)= & h_{0}\left(1+\frac{\delta h(\mathrm{~N})}{h_{0}}-\frac{f\left(\mathrm{M}^{\prime \prime}\right)}{h_{0}}\right. \\
& \left.+\frac{2 R^{2} \eta \sin ^{2}\left(\phi\left(\mathrm{N}, \mathrm{M}^{\prime \prime}\right) / 2\right.}{h_{0}{ }^{2}}+\mathrm{O}\left(10^{-8}\right)\right) \\
r\left(\mathrm{P}^{\prime}, Q\right) & =h_{0}\left(\frac{f\left(\mathrm{M}^{\prime \prime}\right)-z(\mathrm{Q})}{h_{0}}+\mathrm{O}\left(10^{-8}\right)\right)
\end{aligned}
$$

and

$$
\sin \theta\left(\mathrm{H}, \mathrm{P}^{\prime}\right)=\frac{2 R}{h_{0}} \sin \left(\frac{\phi\left(\mathrm{N}, \mathrm{M}^{\prime \prime}\right)}{2}\right)\left(1+\mathrm{O}\left(10^{-4}\right)\right) .
$$

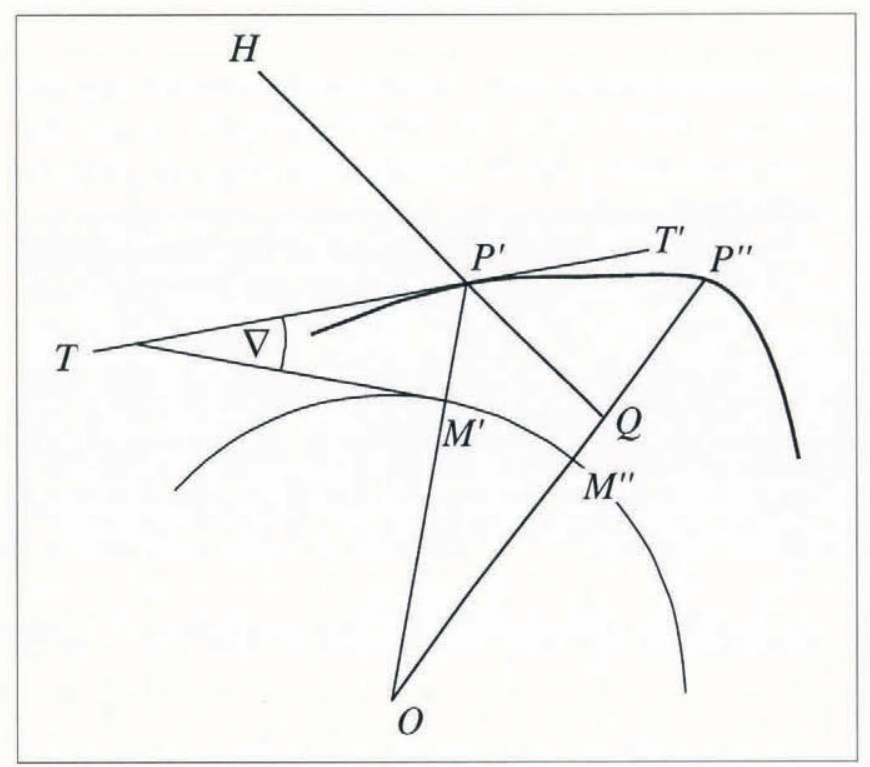

Fig. 2. The approximation to the path lengths $r\left(\mathrm{H}, \mathrm{P}^{\prime}\right)$ and $r\left(\mathrm{P}^{\prime}, \mathrm{Q}\right)$. The location of the point $\mathrm{P}^{\prime}$ is determined implicitly by the location of the point $\mathrm{Q}$ and the shape of the surface. To avoid the difficulties this introduces, the path lengths $r\left(\mathrm{H}, \mathrm{P}^{\prime}\right)$ and $r\left(\mathrm{P}^{\prime}, \mathrm{Q}\right)$ are replaced respectively with the path lengths $r\left(\mathrm{H}, \mathrm{P}^{\prime \prime}\right)$ and $r\left(\mathrm{P}^{\prime \prime}, \mathrm{Q}\right)$. The figure is grossly distorted for clarity.
To sustain Equations (24), (25) and (26), one needs in addition to the approximations we have already made

$$
\sin \phi\left(\mathrm{N}, \mathrm{M}^{\prime}\right)=\sin \phi\left(\mathrm{N}, \mathrm{M}^{\prime \prime}\right)+\mathrm{O}\left(10^{-7}\right)
$$

and

$$
\frac{f\left(M^{\prime}\right)}{h_{0}}=\frac{f\left(\mathrm{M}^{\prime \prime}\right)}{h_{0}}+\mathrm{O}\left(10^{-8}\right) .
$$

For, with the Fresnel approximation, one has

$$
\begin{aligned}
r\left(\mathrm{H}, \mathrm{P}^{\prime}\right)= & h_{0}\left(1+\frac{\delta h(\mathrm{~N})}{h_{0}}-\frac{f\left(\mathrm{M}^{\prime}\right)}{h_{0}}\right. \\
& \left.+\frac{2 R^{2} \eta \sin ^{2}\left(\phi\left(\mathrm{N}, \mathrm{M}^{\prime}\right) / 2\right)}{h_{0}{ }^{2}}+\mathrm{O}\left(10^{-8}\right)\right)
\end{aligned}
$$

and substituting Equations (27) and (28) into Equation (29) then gives Equation (24). Similarly, one has

$$
\begin{aligned}
r\left(\mathrm{P}^{\prime}, \mathrm{Q}\right)= & h_{0}\left(\frac{f\left(\mathrm{M}^{\prime}\right)-z(\mathrm{Q})}{h_{0}}+\frac{2 R^{2} \eta}{h_{0}{ }^{2}}\left(\operatorname { s i n } ^ { 2 } \left(\phi\left(\mathrm{N}, \mathrm{M}^{\prime \prime}\right) / 2\right.\right.\right. \\
& \left.\left.-\sin ^{2}\left(\phi\left(\mathrm{N}, \mathrm{M}^{\prime}\right) / 2\right)\right)+\mathrm{O}\left(10^{-8}\right)\right)
\end{aligned}
$$

and Equation (25) then follows on substituting Equations (27) and (28) into Equation (30). Equation (26) combines the approximation of Equation (22) with Equation (27).

Equation (27) needs the condition Equation (12) that has not been used thus far. To obtain Equation (27), one has on applying the sine rule to the triangle $\mathrm{P}^{\prime} \mathrm{OQ}$

$$
\begin{aligned}
& \sin \left(\phi\left(\mathrm{N}, \mathrm{M}^{\prime \prime}\right)-\phi\left(\mathrm{N}, \mathrm{M}^{\prime}\right)\right) \\
& \quad=\frac{r\left(\mathrm{P}^{\prime}, \mathrm{Q}\right) \sin \left(\theta\left(\mathrm{H}, \mathrm{P}^{\prime}\right)+\phi\left(\mathrm{N}, \mathrm{M}^{\prime}\right)\right)}{R+z(\mathrm{Q})} .
\end{aligned}
$$

$r\left(\mathrm{P}^{\prime}, \mathrm{Q}\right)$ is the path length in the ice whose length is of order $1 / k_{1}$. Using Equations (8) through (12), one finds the quantity on the RHS is zero to order $\mathrm{O}\left(10^{-7}\right)$, which is a better approximation than is needed to sustain Equation (27).

To obtain Equation (28), we suppose that $\mathrm{P}^{\prime}$ is close to $\mathrm{P}^{\prime \prime}$, since Equation (27) tells us that $\phi\left(\mathrm{N}, \mathrm{M}^{\prime \prime}\right)-\phi\left(\mathrm{N}, \mathrm{M}^{\prime}\right)$ is a very small angle, and we assume that the surface can be assumed locally plane between $\mathrm{P}^{\prime}$ and $\mathrm{P}^{\prime \prime}$. Then, applying the sine rule to the triangle $\mathrm{OP}^{\prime} \mathrm{P}^{\prime \prime}$, one has

$$
\frac{R+f\left(\mathrm{P}^{\prime \prime}\right)}{\cos \left(\phi\left(\mathrm{N}, \mathrm{M}^{\prime \prime}\right)-\phi\left(\mathrm{N}, \mathrm{M}^{\prime}\right)+\nabla\right)}=\frac{R+f\left(\mathrm{P}^{\prime}\right)}{\cos \nabla}
$$

Using Equations (13) and (27), one may obtain Equation (28).

One has for the volume element 


$$
\begin{aligned}
\delta V & =\frac{(R+z(\mathrm{Q}))^{2}}{R^{2}} \delta S \delta z(\mathrm{Q}) \\
& =\delta S \delta z(\mathrm{Q})\left(1+\mathrm{O}\left(10^{-4}\right)\right) .
\end{aligned}
$$

The volume integral is bounded above at $z(\mathrm{Q})=f\left(\mathrm{M}^{\prime \prime}\right)$. The exponential loss into the volume allows one to make the lower limit infinite.

Making the approximations, Equations (15), (16), (17), (19), (21), (22), (23), (24), (25), (26) and (33), together with the tidying substitution $\nu=2\left(f\left(\mathrm{M}^{\prime \prime}\right)\right.$ $-z(\mathrm{Q})) / c_{\mathrm{i}}$, Equation (6) takes the form

$$
\begin{aligned}
p(H, t)= & \frac{\lambda^{2} \sigma^{0}}{(4 \pi)^{3} h_{0}^{4}}\left[\iint_{\text {Surface }} \mathrm{d} S g^{2}\left(\frac{2 R}{h_{0}} \sin \left(\frac{\phi(\mathrm{N}, \mathrm{M})}{2}\right)\right)\right. \\
& \cdot\left\{q \left(t-\frac{2}{c}\left(h_{0}+\delta h(\mathrm{~N})-f(\mathrm{M})\right.\right.\right. \\
& \left.\left.+\frac{2 R^{2} \eta \sin ^{2}(\phi(\mathrm{N}, \mathrm{M}) / 2)}{h_{0}}\right)\right) \\
& +\frac{c_{\mathrm{i}} \sigma_{\nu} k_{\mathrm{t}}^{2}}{2 \sigma^{0}} \int_{0}^{\infty} \mathrm{d} \nu \exp \left[-c_{\mathrm{i}} k_{1} \nu\right] \\
& \cdot q\left(t-\nu-\frac{2}{c}\left(h_{0}+\delta h(\mathrm{~N})-f(\mathrm{M})\right.\right. \\
& \left.\left.\left.+\frac{2 R^{2} \eta \sin ^{2}(\phi(\mathrm{N}, \mathrm{M}) / 2}{h_{0}}\right)\right)\right\}
\end{aligned}
$$

to $\mathrm{O}\left(10^{-2}\right)$

\subsection{The measurement interval}

The echo $p(\mathrm{H}, t)$ is a function of time and space. The boundaries of this space are important theoretically; they impact directly on the question of the uniqueness of any deduction from the echo concerning $f$. In practice, these boundaries are determined by a number of complex interactions between the altimeter-control system and the echoes. In this paper, we shall simplify these boundaries with a view to illuminating the uniqueness issue. We shall nonetheless find that the results have important practical implications.

The time origin in Equation (34) is the instant of transmission of the pulse. It is convenient to change this origin by introducing the time-advanced echo

$$
p_{\mathrm{a}}(\mathrm{H}, t)=p\left(\mathrm{H}, t+\frac{2}{c}\left(h_{0}+\delta h(\mathrm{~N})\right)\right) .
$$

This changes the time origin to the instant an echo from the point $\mathrm{N}$ on the reference sphere is received at the altimeter. We shall assume that the time-advanced echoes are known over an interval of time $t \in\left[T_{0}, T_{1}\right]$, where $T_{0}$ and $T_{1}$ are fixed constants.

It is apparent from Equations (34) and (35) that the function $p_{\mathrm{a}}(\mathrm{H}, t)$ is a function that depends on the location of $\mathrm{H}$ only through the location of the point $\mathrm{N}$ on the sphere, and may be written $p_{\mathrm{a}}(\mathrm{N}, t)$. We shall now assume that $p_{\mathrm{a}}(\mathrm{N}, t)$ is known over the entire sphere.

\section{THE INVERSE PROBLEM}

In this section, we are concerned with determining the average height of the surface

$$
\bar{f}=\frac{1}{A} \iint_{\text {Surface }} \mathrm{d} S f(\mathrm{M})
$$

where "Surface" is the projection of the surface on to the sphere, and $A$ is the area of the projection. We can, equally, determine the quantity

$$
\bar{q}(t)=\frac{1}{A} \iint_{\text {Surface }} \mathrm{d} S q\left(t+\frac{2 f(\mathrm{M})}{c}\right) .
$$

With $\bar{q}(t)$ determined, $\bar{f}$ can be calculated. This statement holds, because we may assume without loss of generality (WLOG) that the function $q(t)$ has the properties

$$
\begin{aligned}
& \int_{-\infty}^{\infty} \mathrm{d} t q(t)=1 \\
& \int_{-\infty}^{\infty} \mathrm{d} t t q(t)=0 .
\end{aligned}
$$

Equation (38) holds because $q(t)$ is non-negative and time-limited. The LHS of Equation (38) therefore equals a positive constant. If this constant does not equal unity, one could replace $q(t)$ with a normalized function such that it did. One may assume Equation (39) holds because, if it did not, one could always replace $q(t)$ with a shifted version of itself such that the new function satisfied Equation (39). With Equations (38) and (39), one has

$$
\begin{aligned}
-\frac{c}{2} \int_{-\infty}^{\infty} \mathrm{d} t t \bar{q}(t) & =-\frac{c}{2 A} \iint_{\text {Surface }} \mathrm{d} S \int_{-\infty}^{\infty} \mathrm{d} t t q\left(t+\frac{2 f(\mathrm{M})}{c}\right) \\
& =\frac{1}{A} \iint_{\text {Surface }} \mathrm{d} S f(\mathrm{M})
\end{aligned}
$$

and $\bar{f}$ can be determined from Equation (36).

The average height is to be determined from the measurements $p_{\mathrm{a}}(\mathrm{N}, t): t \in\left[T_{0}, T_{1}\right] ; \mathrm{N} \in$ Sphere. For this purpose, it is better to reformulate the expression for the echo in a way that treats the function $q(t-2 f(\mathrm{M}) / c)$ as the unknown. This is straightforward. With Equations (34), (35) and the relation

$$
q(t)=\int_{\tau=-\infty}^{\infty} \mathrm{d} \tau q(\tau) \delta(t-\tau)
$$

one has 


$$
\begin{aligned}
p_{\mathrm{a}}(\mathrm{N}, t)= & \frac{\lambda^{2} \sigma^{0}}{(4 \pi)^{3} h_{0}{ }^{4}}\left[\int_{\tau=-\infty}^{\infty} \mathrm{d} \tau \iint_{\text {Surface }} \mathrm{d} S g^{2}\left(\frac{2 R}{h_{0}} \sin \left(\frac{\phi(\mathrm{N}, \mathrm{M})}{2}\right)\right)\right. \\
& \cdot\left\{\delta\left(t-\tau-\frac{4 R^{2} \eta \sin ^{2}(\phi(\mathrm{N}, \mathrm{M}) / 2)}{c h_{0}}\right)\right. \\
& +\frac{c_{\mathrm{i}} \sigma_{\nu} k_{\mathrm{t}}{ }^{2}}{2 \sigma^{0}} \int_{0}^{\infty} \mathrm{d} \nu \exp \left[-c_{\mathrm{i}} k_{1} \nu\right] \\
& \left.\cdot \delta\left(t-\tau-\nu-\frac{4 R^{2} \eta \sin ^{2}(\phi(\mathrm{N}, \mathrm{M}) / 2}{c h_{0}}\right)\right\} \\
& \left.q\left(\tau+\frac{2 f(\mathrm{M})}{c}\right)\right] t \in\left[T_{0}, T_{1}\right] ; N \in \text { Sphere. }
\end{aligned}
$$

Equation (42) is a linear, integral equation for $q(t+2 f(\mathrm{M}) / c)$. Clearly, one could deal with the problem by solving Equation (42) for $q(t+2 f(\mathrm{M}) / c)$, and using first Equation (37) and second Equation (40) to determine the average height. This method of solution is certainly of interest if one were concerned with the height of the surface $f$. However, our interest is only in the average height, and the central result of this paper is that a simpler solution is available for the average height. Some practical aspects of solving Equation (42) for the height have been given elsewhere (Wingham and others, 1993).

In this section, we first derive a linear integral equation for $\bar{q}(t)$. The form of this equation allows us to investigate simply sufficient conditions for the unique recovery of the average height from the altimeter echoes. In the remainder of the section, we extend our results to determining the average height of a local region and finding the average height relative to a locally selected datum. These extensions will later allow a relaxation in practice of the criteria for an unique determination of the average height.

\subsection{An integral equation for $\bar{q}(t)$; its solution space}

To obtain an integral equation for $\bar{q}(t)$, we integrate both sides of Equation (42), change the order of the integrations on the RHS, and obtain

$$
\begin{aligned}
\iint_{\text {Sphere }} \mathrm{d} S p_{\mathrm{a}}(\mathrm{N}, t)=\frac{\lambda^{2} \sigma^{0}}{(4 \pi)^{3} h_{0}{ }^{4}} \\
\cdot\left[\int_{-\infty}^{\infty} \mathrm{d} \tau \iint_{\text {Surface }} \mathrm{d} S q\left(\tau+\frac{2 f(\mathrm{M})}{c}\right)\right. \\
\cdot\left\{I(\mathrm{M}, t-\tau)+\frac{c_{\mathrm{i}} \sigma_{\nu} k_{\mathrm{t}}{ }^{2}}{2 \sigma^{0}} \int_{0}^{\infty} \mathrm{d} \nu\right. \\
\left.\left.\cdot I(\mathrm{M}, t-\tau-\nu) \exp \left[c_{\mathrm{i}} k_{\mathrm{t}} \nu\right]\right\}\right] t \in\left[T_{0}, T_{1}\right]
\end{aligned}
$$

where

$$
\begin{aligned}
I(\mathrm{M}, t)= & \iint_{\text {Sphere }} \mathrm{d} S g^{2}\left(\frac{2 R}{h_{0}} \sin \left(\frac{\phi(\mathrm{N}, \mathrm{M})}{2}\right)\right) \\
& \cdot \delta\left(t-\frac{4 R^{2} \eta \sin ^{2}(\phi(\mathrm{N}, \mathrm{M}) / 2}{c h_{0}}\right) .
\end{aligned}
$$

To determine the function $I$, choose a polar coordinate system with an origin at the point $\mathrm{O}$ and an axis of rotation through the point M. Let $\varphi$ and $\rho$ be respectively the polar and azimuthal angles of the point N. One has that

$$
\begin{aligned}
I(\mathrm{M}, t)= & R^{2} \int_{0}^{\pi} \mathrm{d} \varphi \sin \varphi g^{2}\left(\frac{2 R}{h_{0}} \sin \left(\frac{\varphi}{2}\right)\right) \\
& \cdot \delta\left(t-\frac{4 R^{2} \eta \sin ^{2}(\varphi / 2)}{c h_{0}}\right) \int_{0}^{2 \pi} \mathrm{d} \rho \\
= & 2 \pi R^{2} \int_{0}^{\pi} \mathrm{d} \varphi \sin \varphi g^{2}\left(\frac{2 R}{h_{0}} \sin \left(\frac{\varphi}{2}\right)\right) \\
& \cdot \delta\left(t-\frac{4 R^{2} \eta \sin ^{2}(\varphi / 2)}{c h_{0}}\right) \\
= & \frac{\pi c h_{0}}{\eta} \int_{0}^{\frac{4 R^{2} \eta}{c h_{0}}} \mathrm{~d} u g^{2}\left(2 \sqrt{\frac{c u}{4 \eta h_{0}}}\right) \delta(t-u) \\
= & \left\{\begin{array}{cc}
0 & t \notin\left[0,4 R^{2} \eta /\left(c h_{0}\right)\right] \\
\left.\left(\pi c h_{0} / \eta\right)\right) g^{2}\left(\sqrt{c t /\left(\eta h_{0}\right)}\right. & \left.t \in\left[0,4 R^{2} \eta / c h_{0}\right)\right] .
\end{array}\right.
\end{aligned}
$$

Equation (45) gives two pieces of information concerning $I$ which are important to us. First, $I$ is independent of the location of the point $\mathrm{M}$, which is essential to the argument of this section. Secondly, $I$ is a causal, time-limited function. Its causality simplifies the questions of uniqueness we will consider in section 4 .

The time-limiting of $I$ arises because, for a spherical surface, there is a minimum and a maximum interval over which echoes may arrive at the altimeter, corresponding to points on the sphere nearest to, and farthest from the altimeter. The value of the upper limit is incorrect, however, because in section 3.2 we approximated the argument of the $\delta$ function in Equation (44) by assuming that the angle $\phi(\mathrm{N}, \mathrm{M})$ was small, which is not the case for points farthest from the altimeter. The basis for assuming that $\phi(\mathrm{N}, \mathrm{M})$ is small is that the antenna-gain pattern illuminates only a small region of the sphere. Thus, by assumption, the gain function $g^{2}$ in Equation (45) is negligible except for small values of its argument, and one need be concerned only with values of $t$ for which

$$
\sqrt{\frac{c t}{\eta h_{0}}} \sim 10^{-2}
$$

a relation that will be useful later on. Equation (46) is simply a restatement of Equation (9). Equation (46) limits the times of interest to values very much smaller than the upper limit of the time interval in Equation (45), and we will treat the function $I$ as if it were one-sided, rather than time-limited.

The RHS of Equation (43) may now be simplified. One has 


$$
\begin{aligned}
& \frac{\lambda^{2} \sigma^{0}}{(4 \pi)^{3} h_{0}^{4}} \int_{-\infty}^{\infty} \mathrm{d} \tau \iint_{\text {Surface }} \mathrm{d} S q\left(\tau+\frac{2 f(\mathrm{M})}{c}\right) \\
& \cdot\left\{I(\mathrm{M}, t-\tau)+\frac{c_{\mathrm{i}} \sigma_{\nu} k_{t}^{2}}{2 \sigma^{0}} \int_{0}^{\infty} \mathrm{d} \nu I(\mathrm{M}, t-\tau-\nu) \exp \left[-c_{\mathrm{i}} k_{\mathrm{l}} \nu\right]\right\} \\
& =\frac{A \lambda^{2} \sigma^{0}}{(4 \pi)^{3} h_{0}{ }^{4}} \int_{-\infty}^{\infty} \mathrm{d} \tau \bar{q}(\tau) \\
& \cdot\left\{I(t-\tau)+\frac{c_{\mathrm{i}} \sigma_{\nu} k_{\mathrm{t}}{ }^{2}}{2 \sigma^{0}} \int_{0}^{\infty} \mathrm{d} \nu I(t-\tau-\nu) \exp \left[c_{\mathrm{i}} k_{1} \nu\right]\right\} \\
& =\frac{A \lambda^{2} \sigma^{0}}{(4 \pi)^{3} h_{0}{ }^{4}} \int_{-\infty}^{t} \mathrm{~d} \tau \bar{q}(\tau) \\
& \cdot\left\{I(t-\tau)+\frac{c_{\mathrm{i}} \sigma_{\nu} k_{\mathrm{t}}{ }^{2}}{2 \sigma^{0}} \int_{0}^{t-\tau} \mathrm{d} \nu I(t-\tau-\nu) \exp \left[c_{\mathrm{i}} k_{1} \nu\right]\right\}(47)
\end{aligned}
$$

where the second line uses the independence of $I$ on the position of $\mathrm{M}$ and Equation (37), and the third line uses the causality of $I$.

Substitution of Equation (47) into the RHS of Equation (43) gives the desired result. The introduction of some notation, however, allows a more compressed representation. Let us set

$$
\hat{p}(t)=\iint_{\text {Sphere }} \mathrm{d} S_{\mathrm{N}} p_{\mathrm{a}}(\mathrm{N}, t)
$$

on the LHS of Equation (43), and use

$$
\begin{aligned}
k(t) & =\frac{\lambda^{2} \sigma^{0} A}{(4 \pi)^{3} h_{0}{ }^{4}} \\
& \cdot\left\{I(t)+\frac{c_{\mathrm{i}} \sigma_{\nu} k_{\mathrm{t}}{ }^{2}}{2 \sigma^{0}} \int_{0}^{t} \mathrm{~d} \nu \exp \left[-c_{\mathrm{i}} k_{1} \nu\right] I(t-\nu)\right\}
\end{aligned}
$$

to compress the RHS of Equation (47). Then, combining Equations (43), (47), (48) and (49) provides

$$
\hat{p}(t)=\int_{-\infty}^{t} \mathrm{~d} \tau k(t-\tau) \bar{q}(\tau) \quad t \in\left[T_{0}, T_{1}\right]
$$

which is a linear integral equation for $\bar{q}(t)$.

To complete the specification of the problem, we now constrain the interval of the solution of Equation (50). We assume that there are two numbers $f_{0}$ and $f_{1}$ such that, for all points $\mathrm{M} \in$ Surface, $f(\mathrm{M}) \in\left[f_{1}, f_{0}\right]$. Since the pulse $q(t)$ has a duration $T_{\mathrm{p}}$, the function $q(t+$ $2 f(\mathrm{M}) / c$ ) is necessarily zero for times less than $-2 f_{0} / c$ $-T_{\mathrm{p}}$ and greater than $-2 f_{1} / c+T_{\mathrm{p}}$. From Equation (37), this is true too for $\bar{q}(t)$. Denoting these two times $t_{0}$ and $t_{1}$ respectively, the solution interval of Equation (50) is restricted to $t \in\left[t_{0}, t_{1}\right]$.

\subsection{The solution to Equation (50); its uniqueness}

In this section, we consider solutions $\bar{x}(t): t \in\left[t_{0}, t_{1}\right]$ to the equation

$$
\hat{p}(t)=\int_{t_{0}}^{t} \mathrm{~d} \tau k(t-\tau) \bar{x}(\tau) \quad t \in\left[T_{0}, T_{1}\right] .
$$

From Equation (50), this equation admits at least the solution $\bar{q}(t)$, and we need not, therefore, consider the question of existence. However, we need to be assured that $\bar{q}(t)$ is the only solution. In what follows, we shall assume we are working in an $L_{2}$ space, which is sufficient for our purposes.

The uniqueness of a solution to Equation (51) depends, generally, on the relation of the measurement interval $\left[T_{0}, T_{1}\right]$ to the solution interval $\left[t_{0}, t_{1}\right]$ and on the properties of the function $k(t)$.

The simplest result one has is the following. If $T_{1}<t_{1}$, the solution to Equation (51) is not unique. To show this, let $\bar{x}_{1}(t): t \in\left[t_{0}, t_{1}\right]$ be a non-trivial function such that $\bar{x}_{1}(t)=0, t \in\left[t_{0}, T_{1}\right]$. One has

$$
\begin{aligned}
\int_{t_{0}}^{t} \mathrm{~d} \tau k(t-\tau)\left(\bar{q}(t)+\bar{x}_{1}(t)\right)=\int_{t_{0}}^{t} \mathrm{~d} \tau k(t-\tau) \bar{q}(t) \\
\\
=\hat{p}(t) \quad t \in\left[T_{0}, T_{1}\right] \\
\quad t \in\left[T_{0}, T_{1}\right]
\end{aligned}
$$

showing $\bar{q}(t)+\bar{x}_{1}(t): t \in\left[t_{0}, t_{1}\right]$ is a second solution of Equation (51), and the result follows.

Next, consider the case $T_{0}=t_{0}, T_{1}=t_{1}$ so that the measurement and solution intervals are coincident. In this case, the solution $\bar{q}(t)$ to Equation (51) is unique. To show this, consider the equation

$$
\hat{p}^{\prime}(t)=k(0) \bar{y}(t)+\int_{t_{0}}^{t} \mathrm{~d} \tau k^{\prime}(t-\tau) \bar{y}(\tau) \quad t \in\left[t_{0}, t_{1}\right] .
$$

Differentiating both sides of Equation (51) with respect to (WRT) $t$, and setting $T_{0}=t_{0}, T_{1}=t_{1}$ on the RHS, shows that, in this case, any solution $\bar{x}(t): t \in\left[t_{0}, t_{1}\right]$ to Equation (51) is also a solution to Equation (53). Thus, to show that the solution $\bar{q}(t)$ to Equation (51) is unique, it is sufficient to show that Equation (53) has a unique solution. But Equation (53) is a second-kind Volterra equation. The solution

$$
\bar{y}(t)=\frac{p^{\prime}(t)}{k(0)}+\frac{1}{k(0)} \int_{t_{0}}^{t} \mathrm{~d} \tau j(t-\tau) \hat{p}^{\prime}(\tau) \quad t \in\left[t_{0}, t_{1}\right]
$$

exists and is unique (Whittaker and Watson, 1927, p. 221-22). Thus, Equation (51) has the unique solution $\bar{q}(t)$. The function $j(t): t \in\left[0, t_{1}-t_{0}\right]$ in Equation (54) may be determined by successive approximation or other means.

Consider now the possibility that the measured interval lies within the solution interval, that is $T_{0}>t_{0}$, $T_{1}=t_{1}, T_{0}=t_{0}, T_{1}<t_{1}$ or $T_{0}>t_{0}, T_{1}<t_{1}$. In this case, the solution is not unique. We have already established that when $T_{1}<t_{1}$, the solution is not unique, so we need only consider the case $T_{0}>t_{0}, T_{1}=t_{1}$ to establish this 
result. For this, we make use again of the fact that Equation (53) is a second-kind Volterra equation. Let $\hat{s}(t): t \in\left[t_{0}, t_{1}\right]$ be a non-trivial function such $\hat{s}\left(t_{0}\right)=0$, $\hat{s}(t)=0: t \in\left[T_{0}, t_{1}\right]$. The Volterra equation

$$
\hat{s}^{\prime}(t)=k(0) \bar{x}_{3}(t)+\int_{t_{0}}^{t} \mathrm{~d} \tau k^{\prime}(t-\tau) \bar{x}_{3}(\tau) \quad t \in\left[t_{0}, t_{1}\right]
$$

has a solution $\bar{x}_{3}(t): t \in\left[t_{0}, t_{1}\right]$ that exists and is unique. Integrating both sides of Equation (55) over $t \in\left[t_{0}, t_{1}\right]$, one obtains

$$
\hat{s}(t)=\int_{t_{0}}^{t} \mathrm{~d} \tau k(t-\tau) \bar{x}_{3}(\tau) \quad t \in\left[t_{0}, t_{1}\right]
$$

on using the fact that $\hat{s}\left(t_{0}\right)=0$. But when $t \in\left[T_{0}, t_{1}\right]$, Equation (56) becomes

$$
0=\int_{t_{0}}^{t} \mathrm{~d} \tau k(t-\tau) \bar{x}_{3}(\tau) \quad t \in\left[T_{0}, t_{1}\right]
$$

because $\hat{s}(t)=0: t \in\left[T_{0}, t_{1}\right]$. Clearly, there are many functions we could choose for $\hat{s}(t)$. Thus, the homogeneous Equation (57) has non-trivial solutions, and thus Equation (51) has more than one solution.

The results we have obtained are sufficient to reach the main conclusion of this section, which is that the average height of a surface may be determined uniquely from observations of the advanced echo $p_{\mathrm{a}}(\mathrm{N}, t)$ : $\mathrm{N} \in$ Sphere, $t \in\left[T_{0}, T_{1}\right]$, provided the limits of the observation interval satisfy $T_{0} \leq t_{0}, T_{1} \leq t_{1}$. It is worth stressing here that this uniqueness proof arises from the general properties of linear equations; it does not carry over if the assumptions we have made to linearize the problem do not hold in any given situation.

It is not generally possible, however, to state that these conditions on the measured interval are necessary. There exists the possibility that cases for which $T_{0}>t_{0}, T_{1}>t_{1}$ will provide unique solutions to Equation (51). When $T_{0}>t_{0}, T_{1}>t_{1}$, however, the equation analagous to Equation (47) is no longer of the Volterra type, and the situation is not straightforward. The uniqueness or otherwise of solutions to Equation (51) depend on the particular properties of the function $k(t)$ (not on its causality), which in turn depend on the particular form of the antenna pattern, and these have to be investigated on a case-by-case basis. We would remark, however, that for the antenna pattern introduced by Brown (1977), and widely used since, as an approximation to practical altimeter patterns, it turns out that the kernel is separable, and in this case it is easy to demonstrate that the conditions above are sufficient and necessary. (By separable, we mean the kernel $k(t-\tau)$ may be written as the product of a function of $t$ and a function of $\tau$.)

\subsection{Locally averaged topography}

In the previous two sections, we described how the average height of the surface may be determined. It may well be useful, however, to determine the average height of a local region of the surface. This average will obviously depend on the size of the region. As the region becomes very small, the average height will tend to the height itself. In this section, we wish to determine how small the local region can be before the reduction of the problem by integration to a one-dimensional integral equation is no longer possible?

Let $\mathrm{S}$ be a point on the sphere, and let $\phi(\mathrm{S}, \mathrm{M})$ be the angle subtended at the point $\mathrm{O}$ by the lines OS and OM. Let $C \exp \left(-(1-\cos (\phi(\mathrm{S}, \mathrm{M}))) / \tan ^{2}\left(\phi_{\mathrm{W}} / 2\right)\right)$ be a weighting function, where the constant $C$ is chosen to satisfy

$$
\frac{C}{A} \iint_{\text {Surface }} \mathrm{dS} \exp \left(-\frac{1-\cos \phi(\mathrm{S}, \mathrm{M})}{\tan ^{2}\left(\phi_{\mathrm{W}} / 2\right)}\right)=1
$$

When $\phi_{\mathrm{W}}$ is small, the argument of the exponential is large and negative except when $\phi(\mathrm{S}, \mathrm{M})$ is small. In this case, the weighting function behaves as $\exp (-2(\phi(\mathrm{S}, \mathrm{M}) /$ $\left.\left.\phi_{\mathrm{W}}\right)^{2}\right)$. As $\phi_{\mathrm{W}} \rightarrow \pi$, the weighting function tends to unity over the whole sphere. We define the local average of the height to be

$$
\bar{f}_{\mathrm{W}}(\mathrm{S})=\frac{C}{A} \iint_{\text {Surface }} \mathrm{dS} \exp \left(-\frac{1-\cos \phi(\mathrm{S}, \mathrm{M})}{\tan ^{2}\left(\phi_{\mathrm{W}} / 2\right)}\right) f(\mathrm{M})
$$

and one may regard $\bar{f}_{\mathrm{W}}(\mathrm{S})$ as the height averaged over a region around the point $S$, subtending the angle $\phi_{\mathrm{W}}$ at the point $\mathrm{O}$. Corresponding to the function $\bar{q}(t)$ of Equation (37), we introduce the function

$$
\bar{q}_{\mathrm{W}}(t)=\frac{C}{A} \iint_{\text {Surface }} \mathrm{dS} \exp \left(-\frac{1-\cos \phi(\mathrm{S}, \mathrm{M})}{\tan ^{2}\left(\phi_{\mathrm{W}} / 2\right)}\right) q\left(t-\frac{2 f(\mathrm{M})}{c}\right)
$$

from which $\bar{f}_{\mathrm{W}}(\mathrm{S})$ can be determined in the manner of Equation (40). We also introduce the function

$$
\hat{p}_{\mathrm{W}}(\mathrm{S}, t)=\iint_{\text {Sphere }} \mathrm{d} \mathrm{S}_{\mathrm{N}} \exp \left(-\frac{1-\cos \phi(\mathrm{S}, \mathrm{N})}{\tan ^{2}\left(\phi_{\mathrm{W}} / 2\right)}\right) p_{\mathrm{a}}(\mathrm{N}, t)
$$

in place of Equation (48).

We proceed along the same lines as in section 4.1. Equation (43) is replaced by the equation

$$
\begin{array}{r}
\hat{p}_{\mathrm{W}}(\mathrm{S}, t)=\frac{\lambda^{2} \sigma^{0}}{(4 \pi)^{3} h_{0}^{4}}\left[\int_{-\infty}^{\infty} \mathrm{d} \tau \iint_{\text {Surface }} \mathrm{dS} q\left(\tau+\frac{2 f(\mathrm{M})}{c}\right)\right. \\
\cdot\left\{I_{\mathrm{W}}(\mathrm{S}, \mathrm{M}, t-\tau)\right. \\
\left.\left.+\frac{c_{\mathrm{i}} \sigma_{\nu} k_{\mathrm{t}}^{2}}{2 \sigma^{0}} \int_{0}^{\infty} \mathrm{d} \nu I_{\mathrm{W}}(\mathrm{S}, \mathrm{M}, t-\tau-\nu) \exp \left[-c_{\mathrm{i}} k_{1} \nu\right]\right\}\right] \\
t \in\left[T_{0}, T_{1}\right]
\end{array}
$$


where

$$
\begin{aligned}
& I_{\mathrm{W}}(\mathrm{S}, \mathrm{M}, t)=\iint_{\text {Sphere }} \mathrm{dS}_{\mathrm{N}} \exp \left(-\frac{1-\cos \phi(\mathrm{S}, \mathrm{N})}{\tan ^{2}\left(\phi_{\mathrm{W}} / 2\right)}\right) \\
& \cdot g^{2}\left(\frac{2 R}{h_{0}} \sin \left(\frac{\phi(\mathrm{N}, \mathrm{M})}{2}\right)\right) \delta\left(t-\frac{4 R^{2} \eta \sin ^{2}(\phi(\mathrm{N}, \mathrm{M}) / 2}{c h_{0}}\right) .
\end{aligned}
$$

To evaluate $I_{\mathrm{W}}$, choose as before a polar coordinate system with an axis of rotation $\mathrm{OM}$, and let $\varphi$ and $\rho$ be respectively the polar and azimuthal angles of the point $N$. Let $\varphi_{\mathrm{S}}$ and $\rho_{\mathrm{S}}$ be the polar and azimuthal coordinates of the point $\mathrm{S}$. In this system, a standard result from spherical geometry provides

$$
\cos \phi(\mathrm{S}, \mathrm{N})=\cos \varphi_{\mathrm{S}} \cos \varphi+\sin \varphi_{\mathrm{S}} \sin \varphi \cos \left(\rho-\rho_{\mathrm{S}}\right) .
$$

With the help of the integral in section 8.431.3 of Gradsteyn and Ryzhik (1980), one has, on following the steps of Equation (45) and Equation (46), that $I_{\mathrm{W}}$ is a causal function whose value at non-negative times is

$$
\begin{aligned}
I_{\mathrm{W}}(t, \mathrm{~S}, \mathrm{M})= & \frac{\pi c h_{0}}{\eta} g^{2}\left(\sqrt{\frac{c t}{\eta h_{0}}}\right) \exp \left(-\frac{1-\cos \varphi_{\mathrm{S}}}{\tan ^{2}\left(\phi_{\mathrm{W}} / 2\right)}\right) \\
& \cdot I_{0}\left(\frac{\sin \varphi_{\mathrm{S}}}{\tan ^{2}\left(\phi_{\mathrm{W}} / 2\right)} \sqrt{\frac{h_{0} c t}{R^{2} \eta}} \sqrt{1-\frac{h_{0} c t}{4 R^{2} \eta}}\right) \\
& \cdot \exp \left(-\frac{h_{0} c t \cos \varphi_{\mathrm{S}}}{2 R^{2} \eta \tan ^{2}\left(\phi_{\mathrm{W}} / 2\right)}\right)
\end{aligned}
$$

where $I_{0}$ is a Bessel function of imaginary argument. Expanding in Equation (65) the Bessel function, using equation (8.447.1) of Gradsteyn and Ryzhik (1980), and the exponential, in powers of their argument, one has

$$
\begin{aligned}
& I_{0}\left(\frac{\sin \varphi_{\mathrm{S}}}{\tan ^{2}\left(\phi_{\mathrm{W}} / 2\right)} \sqrt{\frac{h_{0} c t}{R^{2} \eta}} \sqrt{1-\frac{h_{0} c t}{4 R^{2} \eta}}\right) \\
& \cdot \exp \left(-\frac{h_{0} c t \cos \varphi_{\mathrm{S}}}{2 R^{2} \eta \tan ^{2}\left(\phi_{\mathrm{W}} / 2\right)}\right) \\
& =1+\frac{1}{4}\left(\frac{h_{0}}{R}\right)^{2}\left(\frac{c t}{\eta h_{0}}\right) \frac{\left(\sin ^{2} \varphi_{\mathrm{S}}-2 \tan ^{2}\left(\phi_{\mathrm{W}} / 2\right) \cos \varphi_{\mathrm{S}}\right)}{\tan ^{4}\left(\phi_{\mathrm{W}} / 2\right)} \\
& +\mathrm{O}\left(\left(\frac{h_{0}}{R}\right)^{3}\left(\frac{c t}{\eta h_{0}}\right)^{2}\right)
\end{aligned}
$$

Using Equations (10) and (46), it is apparent that provided that $\tan \left(\phi_{\mathrm{W}} / 2\right)$ is not smaller than

$$
\tan \left(\phi_{\mathrm{W}} / 2\right) \sim \mathrm{O}\left(10^{-1}\right)
$$

the RHS of Equation (66) is $1+\mathrm{O}\left(10^{-2}\right)$, and therefore that Equation (65) becomes

$$
I_{\mathrm{W}}(\mathrm{S}, \mathrm{M}, t)=\exp \left(-\frac{1-\cos \varphi_{\mathrm{S}}}{\tan ^{2}\left(\phi_{\mathrm{W}} / 2\right.}\right) I(t)\left(1+\mathrm{O}\left(10^{-2}\right)\right) .
$$

Thus, $I_{\mathrm{W}}$ is a causal function whose dependence on the position of the point $\mathrm{M}$ is, to an approximation consistent with those already made, only through the multiplicative weighting function.

Finally, following the remaining steps from Equations (47) through (50), one arrives at

$$
\hat{p}_{\mathrm{W}}(\mathrm{S}, t)=\frac{A}{C} \int_{-\infty}^{t} \mathrm{~d} \tau k(t-\tau) \bar{q}_{\mathrm{W}}(\mathrm{S}, \tau) \quad t \in\left[T_{0}, T_{1}\right]
$$

in place of Equation (50). But for the leading constant, Equation (69) is formally identical to Equation (50) and has the same solution interval. When $T_{0} \leq t_{0}$ and $T_{1} \geq t_{1}$, $\bar{q}_{\mathrm{W}}(\mathrm{S}, t)$ is determined uniquely by Equation (69), and given by the formula

$$
\begin{array}{r}
\bar{q}_{\mathrm{W}}(\mathrm{S}, t)=\frac{C \hat{p}_{\mathrm{W}}^{\prime}(t)}{A k(0)}+\frac{C}{A k(0)} \int_{t_{0}}^{t} \mathrm{~d} \tau j(t-\tau) \hat{p}_{\mathrm{W}}^{\prime}(\mathrm{S}, \tau) \\
t \in\left[t_{0}, t_{1}\right] .
\end{array}
$$

Equation (67) gives an answer to the question with which we opened this section. It shows that, if a local average is formed over an area that subtends at the centre of the Earth an angle very much larger than the angle subtended by the area illuminated by the altimeter - two orders of magnitude larger - the method carries over to local averages. One can, however, argue that Equation (67) is too conservative an estimate. Equation (67) ensures that the approximation of Equation (68) holds for all values of $\varphi_{\mathrm{S}}$. However, the weighting function in Equation (68) is negligible except for values of $\varphi_{\mathrm{S}}$ less than or of the same order as $\varphi_{\mathrm{W}}$. Since we are concerned with small values of $\varphi_{\mathrm{W}}$, one may use $\sin ^{2} \varphi_{\mathrm{W}} \sim 4 \tan ^{2}\left(\phi_{\mathrm{W}} / 2\right)$ in Equation (66), in which case Equation (67) becomes

$$
\tan \left(\phi_{\mathrm{W}} / 2\right) \sim 10^{-2} \text {. }
$$

Equation (71) describes an average formed over an area that subtends at the centre of the Earth an angle an order of magnitude larger than the angle subtended by the area illuminated by the altimeter.

Finally, we remark that we have chosen a particular weighting function so that approximations can be made with a degree of definiteness. We suppose, however, that the result does not depend strongly on this choice, and any smoothly varying function would probably suffice.

\subsection{A locally selected datum}

Thus far, we have restricted our treatment to one in which a single reference sphere is used. We now term this sphere the geocentric sphere. There may be circumstances, however, when it is useful to consider using a spherical datum surface whose origin varies with the region of the local average. We term such a sphere the local sphere. The use of a reference sphere chosen locally complicates matters, because the antenna boresight will not generally pass through the origin of the local sphere. In this section, we extend the method to include this case, assuming that the antenna boresight is held to pass through the origin of the geocentric sphere. 
To form a local sphere, let $\mathrm{O}_{\mathrm{g}}$ lie at the centre of the geocentric sphere, and let SG be a line making an angle $\Delta$ with the tangent plane to the geocentric sphere at $\mathrm{S}$. The arrangement is shown in Figure 3. We shall assume that

$$
\Delta \sim 10^{-1} \text {. }
$$

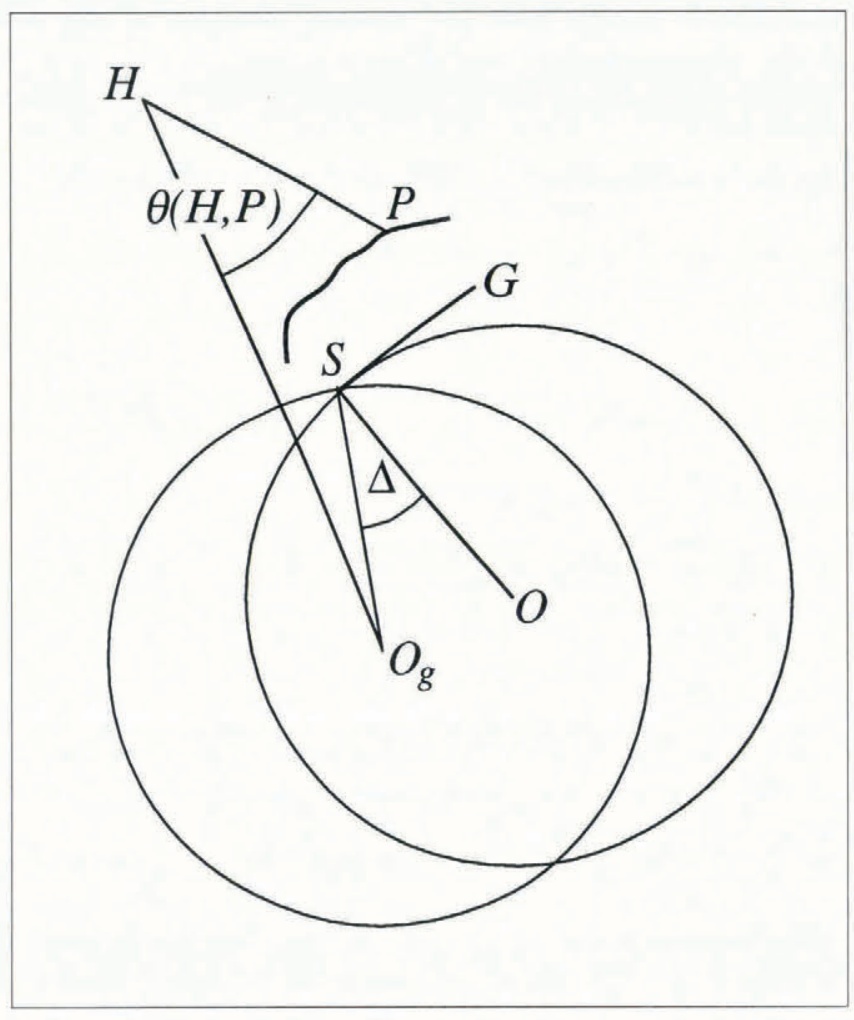

Fig. 3. The formation of a local datum sphere. The sphere is formed by rotating the line $\mathrm{SO}_{\mathrm{g}}$ through an angle $\Delta$ about the point $\mathrm{S}$. The point $\mathrm{G}$, which lies in the tangent plane at the point $\mathrm{S}$ to the local sphere, defines the plane of rotation. In section 5, we will find there are practical advantages in bringing the tangent plane of the local sphere into coincidence with any regional trend in the ice-sheet surface containing the point $\mathrm{P}$. This is illustrated in the figure. The choice of a local datum complicates the problem, because the antenna boresight lies along the line $\mathrm{HO}_{\mathrm{g}}$ and not, as previously, along the line $\mathrm{HO}$. Note that while the points $\mathrm{O}, \mathrm{O}_{\mathrm{g}}, \mathrm{S}$ and $\mathrm{G}$ are co-planar by definition, the points $\mathrm{H}$ and $\mathrm{P}$ generally are not.

The points $\mathrm{O}_{\mathrm{g}}, \mathrm{S}$ and $\mathrm{G}$ define a plane. Rotate within this plane through an angle $\Delta$ the line $\mathrm{O}_{\mathrm{g}} \mathrm{S}$ about $\mathrm{S}$ in such a way that a new line OS is formed normal to the direction of SG. The local sphere is the sphere with centre $\mathrm{O}$ and radius OS; its tangent plane at $\mathrm{S}$ contains $\mathrm{SG}$. All our previous geometric descriptions now apply to the local sphere, so that the point $\mathrm{O}$ now lies at the centre of the local sphere, the points $\mathrm{M}, \mathrm{N}$ and $\mathrm{S}$ now refer to points on the local sphere, and the satellite altitude is assumed equal to the constant $h_{0}$ measured from the local sphere.

We now seek to find the local average height relative to the local sphere. We proceed along the same lines as in the preceding section. The change one needs to make is to replace the function $I_{\mathrm{W}}$ in Equation (63) with the function

$$
\begin{array}{r}
I_{\mathrm{WL}}(\mathrm{S}, \mathrm{M}, t)=\iint_{\text {Sphere }} \mathrm{d} \mathrm{S}_{\mathrm{N}} \exp \left(-\frac{1-\cos \phi(\mathrm{S}, \mathrm{N})}{\tan ^{2}\left(\phi_{\mathrm{W}} / 2\right)}\right) \\
\cdot g^{2}(\sin \theta(\mathrm{H}, \mathrm{P})) \delta\left(t-\frac{4 R^{2} \eta \sin ^{2}(\phi(\mathrm{N}, \mathrm{M}) / 2)}{c h_{0}}\right) .
\end{array}
$$

In the present case, the angle $\theta(\mathrm{H}, \mathrm{P})$, on which $g(\sin (\mathrm{H}, \mathrm{P}))$ depends, is the angle subtended at $\mathrm{H}$ by the points $\mathrm{P}$ and $\mathrm{O}_{\mathrm{g}}$, and not, as previously, the angle subtended at $\mathrm{H}$ by the points $\mathrm{P}$ and $\mathrm{O}$.

We shall assume, for simplicity, that the radii of the local sphere and geocentric sphere are equal. The function $\sin (\mathrm{H}, \mathrm{P})$ can be determined by solving for the triangle $\mathrm{O}_{\mathrm{g}} \mathrm{PH}$. For this purpose, set up a Cartesian coordinate system $\{x, y, z\}$ with an origin at the point $\mathrm{O}$. Let the $z$ axis coincide with the line OS. Let the half-plane $x>0, y=0$ contain the point $\mathrm{O}_{\mathrm{g}}$. Then, the coordinates of the point $\mathrm{O}_{\mathrm{g}}$ are

$$
\left\{R \sin \Delta, 0,2 R \sin ^{2}(\Delta / 2)\right\},
$$

the coordinates of the point $\mathrm{P}$ are

$$
\begin{aligned}
\{ & (R+f(\mathrm{M})) \sin \phi(\mathrm{S}, \mathrm{M}) \cos \rho(\mathrm{S}, \mathrm{M}), \\
& (R+f(\mathrm{M})) \sin \phi(\mathrm{S}, \mathrm{M}) \sin \rho(\mathrm{S}, \mathrm{M}), \\
& (R+f(\mathrm{M})) \cos \phi(\mathrm{S}, \mathrm{M})\}
\end{aligned}
$$

and the coordinates of the point $\mathrm{H}$ are

$$
\begin{aligned}
& \left\{\left(R+h_{0}\right) \sin \phi(\mathrm{S}, \mathrm{N}) \cos \rho(\mathrm{S}, \mathrm{N}),\right. \\
& \left(R+h_{0}\right) \sin \phi(\mathrm{S}, \mathrm{N}) \sin \rho(\mathrm{S}, \mathrm{N}), \\
& \left.\left(R+h_{0}\right) \cos \phi(\mathrm{S}, \mathrm{N})\right\} .
\end{aligned}
$$

In these expressions, $\rho(\mathrm{S}, \mathrm{M})$ and $\rho(\mathrm{S}, \mathrm{N})$ are respectively the angles between the positive $x$ axis and the projection of the lines OM and ON on to the plane $z=0$. With these determinations, the lengths and angles of the triangle may be found with some lengthy, but entirely straightforward, algebra. The result is

$$
\sin \theta(\mathrm{H}, \mathrm{P})=\sqrt{1-\cos ^{2} \theta(\mathrm{H}, \mathrm{P})}
$$

$$
\begin{aligned}
\cos \theta(\mathrm{H}, \mathrm{P}) & =[1-(\kappa(\mathrm{M}) / \eta) \cos (\phi(\mathrm{N}, \mathrm{M})) \\
& -(1 / \eta)\{\Theta(\mathrm{S}, \mathrm{N})-(\kappa(\mathrm{M}) / \eta) \Theta(\mathrm{S}, \mathrm{M})\}] \\
& \cdot\left(1+(\kappa(\mathrm{M}) / \eta)^{2}-2(\kappa(\mathrm{M}) / \eta) \cos (\phi(\mathrm{N}, \mathrm{M}))\right)^{-\frac{1}{2}} \\
& \left.\left.\cdot(1-(2 / \eta) \Theta(\mathrm{S}, \mathrm{N})+4 / \eta)^{2} \sin ^{2}(\Delta / 2)\right)\right)^{-\frac{1}{2}}
\end{aligned}
$$

where

$$
\kappa(\mathrm{M})=\left(1+\frac{f(\mathrm{M})}{R}\right)
$$


with

$$
\begin{aligned}
\Theta(\mathrm{S}, \mathrm{N})= & \sin \Delta \sin \phi(\mathrm{S}, \mathrm{N}) \cos \rho(\mathrm{S}, \mathrm{N}) \\
& +2 \sin ^{2}(\Delta / 2) \cos \phi(\mathrm{S}, \mathrm{N})
\end{aligned}
$$

and $\Theta(S, M)$ is the function obtained by replacing $\mathrm{N}$ in Equation (77) with $\mathrm{M}$.

As previously, one looks for approximations to these functions that will allow one to arrive at a separable form for $I_{\mathrm{WL}}$, similar to Equation (68). However, the smallness of the angles $\phi(\mathrm{N}, \mathrm{M})$ and $\Delta$ are not sufficient for this. One needs in addition to suppose that

$$
\phi(\mathrm{S}, \mathrm{N}) \sim 10^{-1}
$$

Then, with Equations (9), (10), (72) and (78), one has

$$
\begin{aligned}
\sin \theta(\mathrm{H}, \mathrm{P})= & \frac{2 R}{h_{0}}\left[\sin ^{2} \frac{\phi(\mathrm{N}, \mathrm{M})}{2}+\frac{h_{0}^{2}}{R^{2} \eta^{2}} \sin ^{2}\left(\frac{\Delta}{2}\right)\right. \\
& +\frac{h_{0} \sin \Delta}{2 R \eta}(\sin \phi(\mathrm{S}, \mathrm{N}) \cos \rho(\mathrm{S}, \mathrm{N}) \\
& -\sin \phi(\mathrm{S}, \mathrm{M}) \cos \rho(\mathrm{S}, \mathrm{M}))]^{\frac{1}{2}}
\end{aligned}
$$

to $\mathrm{O}\left(10^{-2}\right)$. Considering the integral of Equation (73) for $I_{\mathrm{WL}}$, one sees that this may be justified provided $\tan \left(\phi_{\mathrm{W}} / 2\right)$ is not of a larger order than

$$
\tan \left(\phi_{\mathrm{W}} / 2\right) \sim 10^{-1}
$$

for then the weighting function will ensure that only values of $\phi(\mathrm{S}, \mathrm{N})$ of this order will contribute to the integral. This constraint on $\tan \left(\phi_{\mathrm{W}} / 2\right)$ is consistent with Equations (67) and (71).

Before proceeding, note that if one sets $\Delta=0$ in Equation (73), so that the geocentric and local spheres are coincident and the antenna boresight is normal to their surface, one obtains the result in Equation (22) we have used previously for the argument of the antenna pattern.

To evaluate $I_{\mathrm{WL}}$, choose as before a polar coordinate system with an axis of rotation $\mathrm{OM}$, and let $\varphi$ and $\rho$ be respectively the polar and azimuthal angles of the point $\mathrm{N}$. Let $\varphi_{\mathrm{S}}$ and $\rho_{\mathrm{S}}$ be the polar and azimuthal coordinates of the point $\mathrm{S}$. Let $\rho_{\mathrm{SM}}$ be the angle $\rho(\mathrm{S}, \mathrm{M})$. In this coordinate system, one has with Equations (9), (10) and (78)

$$
\begin{aligned}
& \sin \phi(\mathrm{S}, \mathrm{N}) \cos \rho(\mathrm{S}, \mathrm{N})-\sin \phi(\mathrm{S}, \mathrm{M}) \cos \rho(\mathrm{S}, \mathrm{M}) \\
& =\sin \varphi \cos \left(\rho-\rho_{\mathrm{SM}}\right)+\mathrm{O}\left(10^{-2}\right)
\end{aligned}
$$

$$
\begin{aligned}
& I_{\mathrm{WL}}(\mathrm{S}, \mathrm{M}, t)=R^{2} \int_{0}^{2 \pi} \mathrm{d} \rho \int_{0}^{\pi} \mathrm{d} \varphi \sin \varphi \\
& \cdot \exp \left(-\frac{1-\cos \varphi_{\mathrm{S}} \cos \varphi-\sin \varphi_{\mathrm{S}} \sin \varphi \cos \left(\rho-\rho_{\mathrm{S}}\right)}{\tan ^{2}\left(\phi_{\mathrm{W}} / 2\right)}\right) \\
& \cdot \delta\left(t-\frac{4 R^{2} \eta \sin ^{2}(\varphi / 2)}{\operatorname{ch}_{0}}\right) g^{2}\left(\frac{2 R}{h_{0}}\right. \\
& \left.\cdot \sqrt{\sin ^{2} \frac{\varphi}{2}+\frac{h_{0}^{2}}{R^{2} \eta^{2}} \sin ^{2}\left(\frac{\Delta}{2}\right)+\frac{h_{0}}{2 R \eta} \sin \Delta \sin \varphi \cos \left(\rho-\rho_{\mathrm{SM}}\right)}\right) \\
& =\frac{c h_{0}}{2 \eta} \int_{0}^{2 \pi} \mathrm{d} \rho \\
& \cdot \exp \left(-\frac{1-\cos \varphi_{\mathrm{S}}\left(1-\frac{c h_{0} t}{2 R^{2} \eta}\right)-\sin \varphi_{\mathrm{S}} \sqrt{\frac{c h_{0} t}{R^{2} \eta}} \cos \left(\rho-\rho_{\mathrm{S}}\right)}{\tan ^{2}\left(\phi_{\mathrm{W}} / 2\right)}\right) \\
& \cdot g^{2}\left(\sqrt{\frac{c t}{h_{0} \eta}+\frac{4}{\eta^{2}} \sin ^{2}\left(\frac{\Delta}{2}\right)+\frac{2 R}{h_{0} \eta} \sin \Delta \sqrt{\frac{\operatorname{ch}_{0} t}{R^{2} \eta}} \cos \left(\rho-\rho_{\mathrm{SM}}\right)}\right)
\end{aligned}
$$

to $\mathrm{O}\left(10^{-2}\right)$. For $t \notin\left[0,4 R^{2} \eta /\left(c h_{0}\right)\right], I_{\mathrm{WL}}$ is zero. One now proceeds, as previously, by regarding $I_{\mathrm{WL}}$ as a one-sided function, and using Equation (71) to ignore the time-dependent terms in the exponential. The result is

$$
\begin{gathered}
I_{\mathrm{WL}}(\mathrm{S}, \mathrm{M}, t) \\
=\frac{c h_{0}}{2 \eta} \exp \left(-\frac{1-\cos \varphi_{\mathrm{S}}}{\tan ^{2}\left(\phi_{\mathrm{W}} / 2\right)}\right) \int_{0}^{2 \pi} \mathrm{d} \rho \\
\cdot g^{2}\left(\sqrt{\left.\frac{c t}{h_{0} \eta}+\frac{4}{\eta^{2}} \sin ^{2}\left(\frac{\Delta}{2}\right)+\frac{2 R}{h_{0} \eta} \sin \Delta \sqrt{\frac{c h_{0} t}{R^{2} \eta}} \cos \left(\rho-\rho_{\mathrm{SM}}\right)\right)} \quad t \geq 0 \quad\right. \text { (83) }
\end{gathered}
$$

to $\mathrm{O}\left(10^{-2}\right)$. The integrand in Equation (83) is periodic and the integration is over one period. The integral is therefore independent of $\rho_{\mathrm{SM}}$. One is now free to extend the method. Following the steps of Equations (47) through (50), one arrives at the equation

$$
\hat{p}_{\mathrm{W}}(\mathrm{S}, t)=\int_{-\infty}^{t} \mathrm{~d} \tau k_{\mathrm{WL}}(t-\tau) \bar{q}_{\mathrm{W}}(\mathrm{S}, \tau) \quad t \in\left[T_{0}, T_{1}\right]
$$

where

$$
\begin{aligned}
& k_{\mathrm{WL}}(t)= \\
& \quad \frac{\lambda^{2} \sigma^{0} C}{(4 \pi)^{3} h_{0}{ }^{4}}\left(I_{1}(t)+\frac{c_{i} \sigma_{\nu} k_{\mathrm{t}}{ }^{2}}{2 \sigma^{0}} \int_{0}^{t} \mathrm{~d} \nu \exp \left[-c_{\mathrm{i}} k_{1} \nu\right] I_{1}(t-\tau)\right)
\end{aligned}
$$

and $I_{1}(t)$ is a causal function given by 


$$
\begin{aligned}
& I_{1}(t)=\frac{c h_{0}}{2 \eta} \int_{0}^{2 \pi} \mathrm{d} \rho \\
& \cdot g^{2}\left(\sqrt{\left.\frac{c t}{h_{0} \eta}+\frac{4}{\eta^{2}} \sin ^{2}\left(\frac{\Delta}{2}\right)+\frac{2 R}{h_{0} \eta} \sin \Delta \sqrt{\frac{c h_{0} t}{R^{2} \eta}} \cos \rho\right) t \leq 0 .}\right.
\end{aligned}
$$

Equation (84) has the solution

$$
\begin{array}{r}
\bar{q}_{\mathrm{W}}(\mathrm{S}, t)=\frac{\hat{p}_{\mathrm{W}}^{\prime}(t)}{k_{\mathrm{WL}}(0)}+\frac{1}{k_{\mathrm{WL}}(0)} \int_{t_{0}}^{t} \mathrm{~d} \tau j_{\mathrm{WL}}(t-\tau) \hat{p}_{\mathrm{W}}^{\prime}(\mathrm{S}, \tau) \\
t \in\left[t_{0}, t_{1}\right]
\end{array}
$$

that is unique under the conditions previously discussed.

\section{DISCUSSION}

In this section, we consider the implications of the results of section 4 for the design of experiments to determine the average height. These relate to the collection and to the reduction of observations. At the end of the section, we consider the extent to which present practice is in keeping with the results.

\subsection{Separable estimation of the average height}

This paper distinguishes between the problems of determining the height and the average height of a topographic surface from observations of the radar echo. Comparing Equation (42) with Equation (50), the distinction between these two problems is clear. The former requires the solution of a three-dimensional integral equation but the latter is a one-dimensional integral equation. The distinction does not arise from the general properties of linear equations. The average height can be regarded as a projection of the height the operator in Equation (36) is a projection operator - and it is normally no easier to determine the projection of a solution of a linear equation than the solution itself. The distinction is a special property of the problem, and it arises in part from the Fresnel approximation, but also because the datum surface is spherical. Theoretically, the distinction allows one to deal more simply with the uniqueness question concerning the average height. In practice, the distinction is important because it permits the average height to be determined by separable operations. By separable, we mean they proceed with an operation on the echoes that is independent of the coordinate $t$, followed by an operation that is independent of the coordinate N. Separability is a useful property if a procedure is to be applied to a multi-dimensional set of observations.

To obtain a compact form for these operations, the kernel

$$
J(t)=\delta^{\prime}(t)+j(0) \delta(t)-j^{\prime}(t)
$$

is introduced. For a function $\hat{u}(t) \in\left[t_{0}, t_{1}\right]$, integration by parts provides

$$
\begin{aligned}
\int_{t_{0}}^{t_{1}} \mathrm{~d} \tau & J(t-\tau) \hat{u}(\tau) \\
= & \int_{t_{0}}^{t_{1}} \mathrm{~d} \tau\left(\delta^{\prime}(t-\tau)+j(0) \delta(t-\tau)-j^{\prime}(t-\tau)\right) \hat{u}(\tau) \\
= & \hat{u}^{\prime}(t)+j(0) \hat{u}(t)-[j(t-\tau) \hat{u}(\tau)]_{t_{0}}^{t_{1}}+\int_{t_{0}}^{t} \mathrm{~d} \tau j(t-\tau) \hat{u}^{\prime}(\tau) \\
= & \hat{u}^{\prime}(t)+\int_{t_{0}}^{t} \mathrm{~d} \tau j(t-\tau) \hat{u}^{\prime}(\tau)
\end{aligned}
$$

provided $\hat{u}\left(t_{0}\right)=0$. Since Equation (54) determines $\bar{q}(t)$ uniquely, one has on comparing Equation (89) and Equation (54)

$$
\bar{q}(t)=\frac{1}{k(0)} \int_{t_{0}}^{t_{1}} \mathrm{~d} \tau J(t-\tau) \hat{p}(\tau) \quad t \in\left[t_{0}, t_{1}\right]
$$

provided $\hat{p}\left(t_{0}\right)=0$, which may be assumed WLOG. In the case that a local datum is used, the kernel $J(t)$ is replaced with the kernel $J_{\mathrm{WL}}(t)$ by replacing $j(t)$ in Equation (80) with $j_{\mathrm{WL}}(t)$. Equation (90) is the firstkind form of the solution to the first-kind Equation (50). Using Equations (40) and (36) on the LHS of Equation (90), and Equation (48) on the RHS, one has finally

$$
\bar{f}=-\frac{c}{2 k(0)} \int_{t_{0}}^{t_{1}} \mathrm{~d} t t \int_{t_{0}}^{t_{1}} \mathrm{~d} \tau J(t-\tau)\left\{\iint_{\text {Sphere }} \mathrm{dS} p_{\mathrm{a}}(\mathrm{N}, t)\right\} .
$$

Equation (91) describes separable operations on the echo.

\subsection{Continuity of spatial and temporal coverage}

The question of uniqueness is important in practice. If the average height is not uniquely determined by the observations, a situation exists where the measured echoes are consistent with more than one average height. The importance of section 4.2 is that it provides, for the first time, a clear description of that interval of time over which the echo from a topographic surface uniquely determines the average height. Returning to the definition of the times $t_{0}$ and $t_{1}$ in the paragraph following Equation (50), this description is

$$
T_{0}<\frac{2 f_{0}}{c}-T_{\mathrm{p}} ; \quad T_{1} \geq-\frac{2 f_{1}}{c}+T_{\mathrm{p}}
$$

where the LH hard inequality ensures that $\hat{p}\left(t_{0}\right)=0$ which we required to satisfy Equation (90). The description can be put in simple physical terms if we ignore the contribution of the pulse duration: the average height may be determined uniquely if the echo is measured over a time interval that brackets the vertical extent of the topography.

The extrema of the surface, over the area under 
consideration, defines a volume, and the design of an experiment to determine the average height must aim to provide continuous coverage of the volume. The vertical dimension of the volume maps to a time interval. Continuity of coverage is required in the spatial and temporal senses. Therefore, if one is to use time-gated echoes, it is necessary to have some a priori knowledge of the surface in determining the gating. The more closely the surface can be a priori constrained relative to the datum surface, the shorter the duration of the time gate need be.

\subsection{The size of the local region}

The separable quality of Equation (91) is gained by restricting the problem to that of determining only the average height of the surface. Section 4.3 was concerned with determining the minimum area over which an average height can be determined by a separable operation. It was established there that the method could be extended to local averages, provided the averaging is done over a region large enough. Equation (71) tells us that a region an order of magnitude greater in radius than the area illuminated by the altimeter is sufficient. This is why the qualification "large" is included in the title of this paper.

The limitation on the size of the local region arose from our need to use the approximate Equation (68) for the function $I_{\mathrm{W}}(\mathrm{S}, \mathrm{M}, t)$. Two arguments were used to do this: that the weighting function effectively restricts the angular extent of the function $I_{\mathrm{W}}$ to those points $\mathrm{M}$ near the centre $\mathrm{S}$ of the local region, and that the antenna-gain function limits the temporal extent of $I_{\mathrm{W}}$ to those times $t$ satisfying Equation (46). From Equation (49), one also sees that Equation (46) limits the effective extent of the function $k(t)$. However, there is also a constraint on the time interval over which the average echo $\hat{p}_{\mathrm{W}}(t)$ need be known: the uniqueness constraint Equation (92), which limits our interest to times of order

$$
t \sim 2\left(f_{0}-f_{1}\right) / c .
$$

We therefore need to consider which of the two limitations, Equation (46) or Equation (93), is appropriate to determine the minimum size of the local region.

There are three possibilities one need consider. The first is that $\sqrt{2\left(f_{0}-f_{1}\right) /\left(\eta h_{0}\right)} \ll 10^{-2}$. In this case, the time interval needed to bracket the vertical extent of the topography is very much smaller than the extent of the function $k(t)$. From Equation (69), one sees that Equation (93) is the appropriate limit to use. In this case, the minimum size of the local region is determined by the extent of the topography. This is the situation that arises in ocean altimetry. The second case is that $\sqrt{2\left(f_{0}-f_{1}\right) /\left(\eta h_{0}\right)} \sim 10^{-2}$, in which case Equations (46) and (93) result in the same time interval. The final case is $\sqrt{2\left(f_{0}-f_{1}\right) /\left(\eta h_{0}\right)} \gg 10^{-2}$. Here, Equation (46) is the appropriate choice, since, to the order of approximation we are using, the contribution of the function $k(t)$ to the integral on the RHS of Equation (69) is negligible for times larger than that given by Equation (46). In this case, it is the width of the antenna pattern that limits the minimum size of the local region.

\subsection{The practical usefulness of the local average and local datum}

Local averages allow one to resolve fluctuations in the surface height on scales much larger than the area illuminated by the antenna beam. However, they have other practical uses. First, they permit a relaxing in practice of the uniqueness constraints. The vertical extent of a local region of an ice sheet is typically very much smaller than the vertical extent of the ice sheet as a whole. It is apparent from Equation (59) that the weighting function can be chosen in such a way that the height of the surface a long way distant from the centre of the local region makes a negligible contribution to the local average height. One could take these distant heights to have any value one wishes without significantly altering the problem and, in particular, one could take them to have the same vertical extent as the local region. One may, therefore, with sufficient a priori knowledge, reduce the interval of time over which the echoes are recorded to encompass the range of heights in the locality rather than the range of heights of the ice sheet as a whole.

Secondly, in section 3.3 we assumed that the measurement interval includes the entire sphere. In practice, the spatial-measurement interval will be truncated, for a variety of practical reasons. However, the local average height is determined from the local average of the echoes in Equation (61). The choice of the weighting function allows one to deal with edge effects, arising from the spatial truncation of the measurement interval. Provided one does not seek an average height too close to the edge of the survey region, the weighting function can be used to weight out smoothly the truncation effects.

The use of a local datum may permit a further practical relaxing of the uniqueness constraint. If, within the local region, the vertical extent is dominated by a slowly varying trend, then aligning the tangent plane of the local sphere with the trend may reduce the vertical extent of the surface relative to the datum. With sufficient a priori knowledge of the regional trend, this may further reduce the time interval over which the echo need be measured.

The use of a local datum does have a disadvantage, if local averages are to be further averaged to determine the average height of the ice sheet as a whole. If the local averages are referenced to a common datum, this further averaging is straightforward. If the local averages are referenced to different data (datums!), then this further averaging needs to be done with some care.

\subsection{Comparison with present methods of echo reduction}

At present, altimeter echoes from ice sheets are reduced in two stages. The purpose of the first stage is to reduce the echo to a number that estimates the distance from the altimeter to a point on the surface nearest to the altimeter. The purpose of the second stage is to deduce the shape of the surface from the shape of the surface of nearest range. The first of these stages was performed with a functional termed the "retracker" by Martin and others (1983); the second stage was dealt with by an algorithm 
that calculated a height shift termed the "slope-induced error" by Brenner and others (1983). Other authors have proceeded along similar lines since (e.g. Partington and others, 1989; Remy and others, 1989).

There is, in the theory, a superficial analogy with the retracking procedure. Changing the order of integration in Equation (91), and writing

$$
\bar{f}=\frac{1}{4 \pi R^{2}} \iint_{\text {Sphere }} \mathrm{dS}_{\mathrm{N}} f_{\mathrm{s}}(\mathrm{N})
$$

where the function $f_{\mathrm{s}}(\mathrm{N})$ is

$$
f_{\mathrm{s}}(\mathrm{N})=-\frac{4 \pi c R^{2}}{2 k(0)} \int_{t_{0}}^{t_{1}} \mathrm{~d} t t \int_{t_{0}}^{t_{1}} \mathrm{~d} \tau J(t-\tau) p_{\mathrm{a}}(\mathrm{N}, t)
$$

one finds that Equation (95) is a functional that reduces the echo to a number. However, the quantity $f_{\mathrm{s}}$ bears no simple relation to the first arrival time. Generally, it has no simple relation to the surface height at all. Its use lies solely in the fact that, when averaged over space via Equation (94), the result is equal to the average height, relative to the datum used to determine the kernel $J(t)$.

Because the theory here is a linear one, whereas the retracking procedure is not, a usefully general theoretical comparison of the methods is not possible simply. There are, in addition, theoretical difficulties with the "retracking" and "slope-induced error" procedures. As is well known (see e.g. Robin and others, 1983), there is a uniqueness problem in working with first-arrival times and it is not at all obvious how it is to be closed. Moreover, there are implicit assumptions in the retracking procedure concerning the scales of topography that have not been imposed here, and which have never been demonstrated to be self-consistent. Generally, the solution determined by the algorithms summarized by the terms "retracking" and "slope-induced error" will not be a solution to the problem. It is therefore unlikely that pursuing such a comparison would be a profitable endeavour. One might also add that, when these procedures are used in an experiment, one is faced with the difficulty of showing in a general way that errors that arise from the uniqueness problem, or because the determined solution is not in fact a solution of the problem, are negligible.

\subsection{Limitations}

This paper deals with the problem of altimetry of ice sheets in a linear framework by exploiting the smallness of a number of dimensionless parameters associated with the geometry of satellite altimetry and ice sheets. These parameters and our assumptions concerning their magnitude are laid out in section 3.1 and Equations (8)-(17). We have used the smallness of these parameters to neglect contributions that are $\mathrm{O}\left(10^{-2}\right)$ in the defining expression Equation (6) for the echo. In the practical situation, of course, this may not be sufficient and one may need to check the magnitude of some of the terms we have neglected.

Small surface gradients characterize much of the large ice sheets, but large gradients do occur, particularly at the continental margins, in regions of substantial crevassing and near nunataks. Equation (13) restricts the method to ice sheets with small gradients. We used Equation (13) to reach the approximations of Equations (23) and (28). Other problems will also emerge if one tries to extend the method to larger gradients. Our scattering model is omnidirectional within the range of angles illuminated by the altimeter, and this will not be the case if high surface gradients are present. Small gradients are also implicit in our neglecting the refraction at the air-ice interface. Regions of high gradients should be excluded from the average by use of a weighting function.

The model of penetration we have adopted is a simple one. It is central to the method that the surface- and volume-scattering is laterally constant (vertical variations in volume-scattering may be dealt with by an extension of the description here). Without lateral constancy, the reduction of Equation (45) allowing a separable form is not possible. It should be kept in mind that, since an altimeter averages the power scattered from a region some kilometres in lateral extent, it is the volume-scattering averaged on this scale that matters in this context. Variations on scales much larger than this can be dealt with by an appropriate normalization; variations on scales much shorter than this will be invisible to the altimeter. In my experience, this is a good model for the altimeter echoes from the dry cold surface experienced, for example, over much of the Antarctic continent. Over the warmer areas of the Greenland ice sheet, however, the situation may be considerably more complex. Theoretically, the introduction of an unknown lateral variation in scattering coefficients makes the problem non-linear and difficult.

Section 4.4 dealt with a case in which the antenna boresight was not aligned with the normal to the reference sphere. It is important to stress, however, that section 4.4 does not provide an argument that deals with the most general case of a small "mispointing" angle between the antenna boresight and the surface normal to the sphere, which may arise as a result of pitch or roll of the antenna. Moreover, I suspect that generally such an extension is not possible. This is because the echo over a topographic surface is sensitive to the yaw angle of the antenna. In the case dealt with here, this angle is constrained such that the boresight is sighted at a fixed point in space - the centre of the geocentric sphereand this constraint was not added for convenience. It ensures a very particular behaviour of the yaw angle. It is only in this circumstance, combined with a limited local region, that our results hold.

\section{GONGLUSIONS}

This paper is concerned with the altimetry of surfaces with gentle undulations having a wide range of spatial scales that includes the diameter of the area illuminated by the altimeter beam. The aim of the work is to deal with the four questions raised in the introduction: What is the relation between the altimeter echo and the height of a topographic surface? Can a method be found for the inversion of this relationship for the average height? In 
what circumstances is the result of this inversion unique? What do the answers to these questions imply for the design of an experiment to determine the average height of ice sheets?

The general relationship between the surface height and the echo is complicated and non-linear. The geometry of satellite-altimetry operation and the smallness of ice-sheet gradients can be used to give an approximate form for the echo in which the surface height appears only as a linear combination in the argument of the pulse function. With this form, the problem of determining the average height of the surface from observations of the echo can be placed within the framework of a linear inverse problem. Methods for the solution of such problems are well known. The average height determined from the solution of the inverse problem is unique, provided the echo is observed over a sufficient interval of time. The area over which the average is formed may vary but it must be large in comparison with the area illuminated by the altimeter.

These results have implications for the collection and reduction of the echoes in an experiment to determine the average height of a surface. The problem of determining the average height is practically simpler than the problem of determining the height. The average height can be determined by operating on the echo with the kernel resulting from the solution of a one-dimensional integral equation. Continuity of spatial and temporal coverage is needed to ensure a unique determination of the average height and this requires a priori knowledge of the extrema of the surface. This interval may be reduced with greater a priori knowledge of the surface by minimizing the area of the average or by selecting a datum surface aligned with a regional trend in the surface.

This paper does not give a discrete representation that would be needed for an experimental implementation of the theory. There are substantial issues associated with the transfer to a discrete representation. While the inverse problem described here is well posed, it does not follow that it is well conditioned. Numerical stability may need attention. The spatial sampling of the echoes is irregular and errors will arise in the quadrature of a spatial average. This raises the question of what is a sufficient density of spatial sampling? I shall deal with these matters in a separate paper.

\section{ACKNOWLEDGEMENTS}

A number of people made very useful contributions to the evolution of this paper. K. Jansons, Department of Mathematics, University College London, pointed out to me the usefulness of weighted averages. Professor S. Quegan, University of Sheffield, the reviewers and the editor made useful criticisms. The work was in part supported by the U.K. Natural Environment Research Council, under grant GR3/8748.

\section{REFERENCES}

Abramowitz, M.A. and I. Stegun. 1965. Handbook of mathematical functions. New York, Dover Publications.

Berry, M. V. 1973. The statistical properties of echoes diffracted from rough surfaces. Philos. Trans. R. Soc. London, Ser. A, 273, 611-654.

Brenner, A. C., R. A. Bindschadler, R.H. Thomas and H.J. Zwally. 1983. Slope-induced errors in radar altimetry over continental ice sheets. 7. Geophys. Res., 88(C3), 1617-1623.

Brown, G.S. 1977. The average impulse response of a rough surface and its implications. IEEE Trans. Antennas Propag., AP-25, 67-74.

Collin, R. E. and F.J. Zucker. 1969. Antenna theory. Part I. New York, McGraw Hill.

Gradsteyn, I.S. and I. M. Ryzhik. 1980. Tables of integrals, series, and products. San Diego, CA, Academic Press.

Martin, T. V., H.J. Zwally, A. C. Brenner and R.A. Bindschadler. 1983. Analysis and retracking of continental ice sheet radar altimeter waveforms. 7. Geophys. Res., 88(C3), 1608-1616.

Partington, K. C., J. K. Ridley, C. G. Rapley and H.J. Zwally. 1989. Observations of the surface properties of the ice sheets by satellite radar altimetry. f. Glaciol., 35(120), $267-275$.

Remy, F., P. Mazzega, S. Houry, C. Brossier and J. F. Minster. 1989. Mapping of the topography of continental ice by inversion of satellite-altimeter data. f. Glaciol., 35(119), 98-107.

Ridley, J.K. and K. C. Partington. 1988. A model of satellite radar altimeter return from ice sheets. Int. 7. Remote Sensing, 9(4), 601-624.

Robin, G. de Q., D.J. Drewry and V.A. Squire, 1983. Satellite observations of polar ice fields. Philos. Trans. R. Soc. London, Ser. A, 309 (1508), 447-461.

Whittaker, E. T. and G.N. Watson. 1927. A course of modern analysis. Fourth edition. Cambridge, Cambridge University Press.

Wingham, D.J., C. G. Rapley and J.G. Morley. 1993. Improved resolution ice sheet mapping with satellite radar altimeters. EOS 74 $(10), 113,116$ 